\title{
On the time-dependent description for the decay of unstable D-branes
}

\author{
K,Graham ${ }^{1}$, A. Konechny ${ }^{2}$ and J. Teschner ${ }^{3}$ \\ ${ }^{1}$ Institut für theoretische Physik, Freie Universität Berlin, Arnimallee 14, 14195 Berlin, Germany \\ ${ }^{2}$ Department of Physics and Astronomy, Rutgers, The State University of New Jersey \\ Piscataway, NJ 08854, USA \\ ${ }^{3}$ DESY Theory, Notkestr. 8522607 Hamburg Germany
}

\begin{abstract}
We discuss how to describe time-dependent phenomena in string theory like the decay of unstable D-branes with the help of the world-sheet formulation. It is shown in a nontrivial well-controlled example that the coupling of the tachyons to propagating on-shell modes which escape to infinity can lead to time-dependent relaxation into a stationary final state. The final state corresponds to a fixed point of the RG flow generated by the relevant field from which the tachyon vertex operator is constructed. On the way we set up a fairly general formalism for the description of slow time-dependent phenomena with the help of conformal perturbation theory on the world-sheet.
\end{abstract}

\section{Introduction}

Time-dependent phenomena in string theory are not easy to understand. Tractable examples of time-dependent backgrounds are rare, making it hard to extract general lessons about how string theory differs from ordinary field theory in the description of time-dependent phenomena. Conceptual and technical issues appear to be intertwined in a complicated way: If one tries to construct the conformal field theories (CFTs) describing time-dependent backgrounds using nonlinear sigma models, one has to face the unboundedness of the sigma model path integral due to the indefinite signature of the metric. The very definition of CFTs describing timedependent backgrounds is therefore problematic in general. On the other hand, experience from point-particle field theories suggests the definition of the vacuum of the string (field) theory becomes ambiguous in generic time-dependent backgrounds. If and how such technical and conceptual problems are intertwined is not well understood.

An interesting class of time-dependent phenomena is often referred to as tachyon condensation. This includes decay processes of unstable D-branes, interpreted as the condensation of an open string tachyon on the relevant brane. In order to bypass the technical difficulties involved in the definition of a CFT which describes a tachyon condensation process it is often proposed that one can simply restrict attention to the CFT describing the spatial part of the background. 
Tachyons are then described by relevant fields in this CFT. Perturbing the CFT by these relevant fields will generate nontrivial renormalization group (RG) flows, whose end-points are then conjecturally interpreted as the possible final states of decay processes in the genuinely time-dependent description.

The scope and limitations of this picture are not well understood. A major puzzle stems from the fact that the evolution equations for background fields like the tachyon are second order in time derivatives, whereas the RG flow equations are first order differential equations. This seems to exclude any simple relationship between renormalization group flows and the true time evolution. One may still hope the time-independent description using RG flows correctly describes at least certain qualitative features of the true time evolution like the initial and final states. However, the fact that the time evolution equations are second order differential equations suggests that the solutions which describe rolling from an unstable maximum of the tachyon potential will typically exhibit oscillatory behavior and may not relax to any stationary final state as suggested intuitively by the time-independent picture in terms of RG flows.

A first step towards the resolution of this puzzle was recently made in [FHL]. Proper inclusion of the dilaton typically produces a damping force that may lead to relaxation into the minimum of the tachyon potential which corresponds to the end-point of an RG flow in the time-independent description. However, the mechanism studied in [FHL] applies only to supercritical backgrounds.

In the present paper we will propose a different mechanism which reconciles - qualitatively, for a certain class of backgrounds - the time-dependent and the time-independent pictures. The mechanism applies to backgrounds in which a localized tachyonic excitation couples to a continuum of string modes which can escape to infinity. The energy released in the condensation of the localized tachyon is radiated away to infinity. Having infinitely many degrees of freedom into which the energy is dissipated avoids any oscillatory nature for the resulting dynamics of the tachyon.

The noncompactness of the string background is crucial for this mechanism. If the (open) string spectrum is discrete, as is typically the case for compact backgrounds (branes), it may not be true that the system relaxes into a new stationary state.

\section{On the use of conformal perturbation theory.}

It is not trivial to construct examples which illustrate the points above within the world-sheet approach to string theory. The examples we will discuss in this paper will be constructed by means of conformal perturbation theory. However, the application of conformal perturbation theory turns out to be somewhat subtle for this type of problem.

Conformal perturbation theory will be useful in the present context only if there is a parameter $\delta$ in the theory which allows one to make the decay process arbitrarily slow. The parameter $\delta$ is related to the deviation from marginality in the time-independent picture. It also controls the amount of energy stored in the unstable brane. One ultimately aims to develop a series expansion in the parameter $\delta$. 
In some cases we will be dealing with examples where, strictly speaking, no renormalization of the perturbation Lagrangian is necessary. This means the bare coupling constant $\lambda$ is well-defined, and the series expansion in $\lambda$ is meaningful. However, the expansion in $\lambda$ is not useful to extract the corrections to leading order in $\delta$. The use of renormalization group (RG) technology will prove crucial in order to extract these contributions to string emission amplitudes. We will take advantage of the fact that using effective coupling constants as determined by the renormalization group equations effectively amounts to resumming certain contributions to a "naive" perturbative expansion. The fact itself is certainly known from other quantum field theoretical models, but crucial for our present investigation is a more precise statement: Any "proper" regularization scheme 1 leads to the definition of renormalized coupling constants $\lambda_{\text {ren }}$ which themselves are of the order $\delta$, and which allow us to capture the leading order (in $\delta$ ) effects in the first order of perturbation theory in $\lambda_{\text {ren }}$. This result may be known, but at least in the context of conformal perturbation theory we did not find a sufficiently detailed discussion of it in the literature 2. We have therefore included a self-contained discussion of this point in our paper.

A second subtlety is the following. If one uses conformal perturbation theory one might be tempted to drop irrelevant fields from the perturbation Lagrangian. This turns out to lead to incorrect results in our example. Irrelevant fields that are sufficiently close to marginality produce important contributions to correlation functions. In our case they precisely take care of the radiation into strings that propagate out to infinity.

\section{The model.}

The model which will illustrate the mechanism proposed above in a controlled way is the socalled $c=1$ noncritical string theory, see e.g. [GM, $[\mathbf{S S}]$ for reviews. The $c=1$ string theory is a two-dimensional string background with coordinates $\left(X_{0}, \phi\right) \in \mathbb{R}^{2}$, where $X_{0}$ represents time. This background is characterized by the following expectation values for the target-space metric $G_{\mu \nu}$, the dilaton $\Phi$ and the tachyon field $T$ :

$$
G_{\mu \nu}=\eta_{\mu \nu}, \quad \Phi=\phi, \quad T=\mu e^{2 \phi} .
$$

The worldsheet-description of this background is defined by a (boundary) CFT with central charge $c=26$ which is the product of Liouville theory with the CFT of a free timelike boson.

Note the linear growth of the dilaton in (1.1) implies the string coupling is strong for $\phi \rightarrow \infty$, whereas it is weak for $\phi \rightarrow-\infty$. The tachyon expectation value $T=\mu e^{2 \phi}$ produces a force on the closed strings which exponentially grows for $\phi \rightarrow \infty$, and which therefore effectively confines the closed strings to the weak coupling region $\phi \rightarrow-\infty$. This force implies that the closed string states which for early times are injected into the weak coupling region with positive momentum will ultimately be reflected back into the weak coupling region.

\footnotetext{
${ }^{1}$ See Section 2 for the explanation of what we call a proper regularization scheme.

${ }^{2}$ See, however, $[\mathrm{CL}]$ for some remarks in this direction
} 
An open string sector can be introduced by imposing Neumann-type boundary conditions for both the space and the time directions that may include a space-dependent force on the endpoints of the open strings. The corresponding D-branes are often called FZZT branes. These branes are described by a single parameter $\delta$ which may be thought of as a parametrization for the force on the end points of the open strings. It should be emphasized, however, that we are dealing with a case in which semiclassical reasoning is not applicable, and where the stringy corrections are substantial. What can be deduced from the exact solution of boundary Liouville theory [ [FZZ, T1, Ho, $\overline{\mathrm{PT}}, \mathbf{\mathrm { T } 2}]$ is the following: The open string spectrum always contains propagating open strings which bounce off the potential wall coming from the tachyon condensate $T=\mu e^{2 \phi}$. However, for certain values of the parameter $\delta$ the string spectrum also contains bound states. In the case considered in this paper there is a single bound state $|\vartheta\rangle\rangle$ which turns out to be tachyonic. The wave-function of $|\vartheta\rangle\rangle$ decays exponentially towards the weak coupling region. This means the FZZT brane is carrying a localized open string tachyon.

It is natural to interpret the bound state as the result of a balance between an attractive force on the end-points of the strings and the repulsive force acting on the bulk of the string. The very existence of the bound state is an indication of the presence of a sink in some effective potential for open strings on the FZZT branes. We expect the condensation of the open string tachyon $|\vartheta\rangle\rangle$ may "fill up" this sink, producing an FZZT brane on which the forces on the open strings are effectively repulsive. The energy released in this process will be radiated away into the weak coupling region as open and closed strings. We will show this is precisely what happens by constructing a time-dependent solution to noncritical string theory describing tachyon condensation on FZZT branes. One should note that the effect of closed string radiation will be subleading in the string coupling constant $g_{s}$ in the model we will study.

\section{Content of the paper}

The main technical result of the paper is the perturbative construction of a time-dependent solution of noncritical open-closed string theory to lowest order in $\delta$. This will heavily exploit the RG improvement to the perturbative expansion. We will therefore start in section 2 by explaining how RG techniques manage to resum the contributions to leading order in $\delta$.

In the following section we discuss the construction of general time-dependent backgrounds describing open string tachyons. In so doing we will see some of the limitations of timeindependent RG flows as a description of time-dependent phenomena.

Section 4 introduces the relevant aspects of boundary Liouville theory, somewhat sharpening the physical picture of the D1 branes along the way. We also discuss a renormalization group flow obtained by perturbing Liouville theory with a relevant boundary field that provides a first hint towards the scenario discussed in the introduction. The end-point of the RG flow which starts from the boundary condition $\delta$ is identified with the boundary condition associated to the parameter $-\delta$.

Section 5 then discusses the perturbative construction of a time-dependent solution of string theory which is associated to the condensation of the open string tachyon on the D1 branes. It 
is found that the time dependent solution smoothly interpolates between the static solution with parameter $+\delta$ in the asymptotic past and a background which can be seen as the static solution with parameter $-\delta$ plus a propagating radiation background in the infinite future.

Section 6 contains a discussion of the results obtained in the paper and directions for future work.

Appendix A discusses RG equations in the presence of UV divergences which appear when the identity operator is present in the OPE's of perturbing operators. Appendix B contains technical details regarding the boundary Liouville theory.

\section{Renormalization group improvement of perturbative series}

To begin with, we will consider the perturbation of a given boundary conformal field theory (BCFT) by a relevant boundary field with conformal dimension $1-\delta$. Our aim is to show how renormalisation group (RG) technology provides an efficient tool to extract the leading behaviour of correlation functions for $\delta \rightarrow 0$.

To explain the method, we will first consider cases in which all short distance singularities are integrable, so no renormalization is necessary. RG technology is nevertheless found to be an efficient tool for reaching our aim. The existence of divergencies in the perturbative integrals does not change any relevant feature of the resulting picture, as shown in appendix A.

We will consider a BCFT which has a family of boundary conditions $B_{\delta}$ parametrized by a parameter $\delta$. The BCFT under consideration will contain primary boundary fields $\phi_{i}(\tau)$ of conformal weight $h_{i}$, living on the unit circle, $0 \leq \tau<2 \pi$, The labels $i$ take values in some set $F$ (in the examples to follow, $F$ may be a continuum).

To apply perturbation theory usefully, we will assume that the boundary conditions $B_{\delta}$ are parametrized by $\delta$ in such a way that a small value for $\delta$ implies a relevant boundary field $\phi_{0}$ is nearly marginal in the sense that $y_{0} \equiv 1-h_{0}=\mathcal{O}(\delta)$. It will also be important to consider all the other nearly marginal fields, including those which are irrelevant. We will denote this set by $M \subset F$, the elements $\phi_{i}(\tau)$ of $M$ satisfy $y_{i}=1-h_{i}=\mathcal{O}(\delta)$. For simplicity we assume all other fields have $y_{i}=1-h_{i}=\mathcal{O}(1) \cdot 3$

The correlation functions of fields $\phi_{i}(\tau)$ can be evaluated using their OPE, valid for $0<\tau<$ $\pi$

$$
\phi_{i}(\tau) \phi_{j}(0)=\sum_{k \in F} C_{i j}^{k}\left(2 \sin \frac{\tau}{2}\right)^{-1+y_{i}+y_{j}-y_{k}} \phi_{k}(0)+\text { descendents } .
$$

Note that if $F$ contains a continuum, the sum will become an integral. For simplicity, we also assume the OPE coefficients have a nice $\delta$-dependence like $C_{i j}{ }^{k}=\mathcal{O}(1)$.

We will consider a perturbation of the boundary condition by an operator $\phi_{0}(\tau), 0 \in M$,

$$
S_{\text {pert }}=\lambda \int_{\partial \Sigma} d \tau \phi_{0}(\tau)
$$

\footnotetext{
${ }^{3}$ These assumptions, together with a later one concerning the OPE coefficients, can be weakened or adjusted. In fact, in our string theory example $y_{i}=\mathcal{O}\left(\delta^{2}\right)$ and $C_{i j}{ }^{k}=\mathcal{O}(\delta)$. It takes a moment to see such changes do not affect the conclusions.
} 
which defines perturbed correlation functions via

$$
\begin{aligned}
\langle\ldots\rangle_{\lambda} & =\left\langle\ldots e^{-S_{\text {pert }}}\right\rangle_{B_{\delta}} \\
& =\langle\ldots\rangle_{B_{\delta}}-\lambda \int_{\partial \Sigma} d \tau\left\langle\ldots \phi_{0}(\tau)\right\rangle_{B_{\delta}}+\frac{\lambda^{2}}{2} \int_{\partial \Sigma \times \partial \Sigma} d \tau_{1} d \tau_{2}\left\langle\ldots \phi_{0}\left(\tau_{1}\right) \phi_{0}\left(\tau_{2}\right)\right\rangle_{B_{\delta}}+\ldots
\end{aligned}
$$

In the examples to follow, this perturbation will be UV finite and so the coupling constant $\lambda$ is well defined and the perturbation series in $\lambda$ does not require any renormalisation. Our task for this section is to calculate the leading $\delta$ behaviour of this series. We will find that RG technology provides the answer but to see why, it helps to attempt to extract the leading $\delta$ behaviour by brute force.

\subsection{Brute force calculation}

We concentrate on the perturbation expansion (2.3) in the particular case where dots denote the insertion of operators into the interior of the disc. We proceed by taking the $\lambda$ series (2.3) and studying the small $\delta$ behaviour of each term. The $n$th term will be found to go as $\lambda^{n} \delta^{1-n}$. If $\lambda=\mathcal{O}(\delta)$ it is clear the leading correction is $\mathcal{O}(\delta)$, however every order makes a contribution. Our task is to calculate these contributions.

Consider the second order term of (2.3). As $\delta \rightarrow 0$, this term becomes divergent due to the singularity in the OPE (2.1). To extract this singular behaviour, we introduce a distance $L$ inside which the boundary-boundary OPE is valid (this distance will depend on the location of the interior operators). We write,

$$
\frac{\lambda^{2}}{2} \int_{\partial \Sigma} d \tau_{1} \int_{\partial \Sigma} d \tau_{2}\left\langle\ldots \phi_{0}\left(\tau_{1}\right) \phi_{0}\left(\tau_{2}\right)\right\rangle_{B_{\delta}}=\frac{\lambda^{2}}{2} \int_{\left|\tau_{1}-\tau_{2}\right|<L} d \tau_{1} d \tau_{2} \ldots+\frac{\lambda^{2}}{2} \int_{\left|\tau_{1}-\tau_{2}\right|>L} d \tau_{1} d \tau_{2} \ldots
$$

and the first term becomes,

$$
\begin{aligned}
\frac{\lambda^{2}}{2} \int_{\left|\tau_{1}-\tau_{2}\right|<L} d \tau_{1} d \tau_{2}\left\langle\ldots \phi_{0}\left(\tau_{1}\right) \phi_{0}\left(\tau_{2}\right)\right\rangle_{B_{\delta}} \\
=\lambda^{2} \sum_{k \in F} C_{00}{ }^{k} \int_{0}^{L} d u\left(2 \sin \frac{u}{2}\right)^{-1+2 y_{0}-y_{k}} \int_{\partial \Sigma} d \tau\left\langle\ldots \phi_{k}(\tau)\right\rangle_{B_{\delta}}+\text { descendents } \\
=\lambda^{2} \sum_{k \in M} C_{00}{ }^{k} \frac{1}{2 y_{0}-y_{k}} \int_{\partial \Sigma} d \tau\left\langle\ldots \phi_{k}(\tau)\right\rangle_{B_{\delta}}+\text { subleading in } \delta
\end{aligned}
$$

The divergence is independent of our choice of $L$ and comes from the fusion of the perturbation into almost marginal fields (the fields with $y_{i}=\mathcal{O}(\delta)$, both relevant and irrelevant). The other fields contribute to a higher order in $\delta$. Also note that the geometry of the boundary is largely immaterial for this calculation, only the short distance behaviour of the OPE is important. Finally, second term in (2.4) is finite as $\delta \rightarrow 0$ and so represents a subleading contribution to the correlation function. 
Moving to third order, the leading $\delta$ behaviour will come from the region of integration where all the boundary fields come together simultaneously. We again introduce a distance $L$ to isolate this contribution and use the OPE to evaluate the integrals. Note the OPE is only valid for $\tau>0$ so one must be careful:

$$
\begin{aligned}
-\frac{\lambda^{3}}{6} \int_{\left|\tau_{i}-\tau_{j}\right|<L} d \tau_{1} d \tau_{2} d \tau_{3}\left\langle\ldots \phi_{0}\left(\tau_{1}\right) \phi_{0}\left(\tau_{2}\right) \phi_{0}\left(\tau_{3}\right)\right\rangle_{B_{\delta}} \\
=-\lambda^{3} \int d \tau \int_{0}^{L} d u_{1} \int_{u_{1}}^{L} d u_{2}\left\langle\ldots \phi_{0}\left(\tau+u_{2}\right) \phi_{0}\left(\tau+u_{1}\right) \phi_{0}(\tau)\right\rangle_{B_{\delta}} \\
=-\lambda^{3} \int d \tau \int_{0}^{\frac{1}{2} u_{2}} d u_{1} \int_{0}^{L} d u_{2} \ldots-\lambda^{3} \int d \tau \int_{\frac{1}{2} u_{2}}^{u_{2}} d u_{1} \int_{0}^{L} d u_{2} \ldots
\end{aligned}
$$

Applying the OPE in the first term,

$$
\begin{gathered}
-\lambda^{3} \sum_{k, \ell \in S} C_{00}{ }^{k} C_{0 k} \int^{\ell} d \tau \int_{0}^{\frac{1}{2} u_{2}} d u_{1} \int_{0}^{L} d u_{2}\left(2 \sin \frac{u_{1}}{2}\right)^{-1+2 y_{0}-y_{k}}\left(2 \sin \frac{u_{2}}{2}\right)^{-1+y_{0}+y_{k}-y_{\ell}}\left\langle\ldots \phi_{\ell}(\tau)\right\rangle_{B_{\delta}} \\
\quad+\text { descendants } \\
=-\lambda^{3} \sum_{k, \ell \in M} \frac{C_{00}{ }^{k} C_{0 k}{ }^{\ell}}{\left(2 y_{0}-y_{k}\right)\left(3 y_{0}-y_{\ell}\right)} \int d \tau\left\langle\ldots \phi_{\ell}(\tau)\right\rangle_{B_{\delta}}+\text { subleading in } \delta,
\end{gathered}
$$

while the second term gives,

$$
\begin{gathered}
-\lambda^{3} \sum_{k, \ell \in S} C_{00}{ }^{k} C_{k 0}{ }^{\ell} \int d \tau \int_{\frac{1}{2} u_{2}}^{u_{2}} d u_{1} \int_{0}^{L} d u_{2}\left(2 \sin \frac{u_{2}-u_{1}}{2}\right)^{-1+2 y_{0}-y_{k}}\left(2 \sin \frac{u_{1}}{2}\right)^{-1+y_{0}+y_{k}-y_{\ell}}\left\langle\ldots \phi_{\ell}(\tau)\right\rangle_{B_{\delta}} \\
\quad+\text { descendants } \\
=-\lambda^{3} \sum_{k, \ell \in M} \frac{C_{00}{ }^{k} C_{k 0}{ }^{\ell}}{\left(2 y_{0}-y_{k}\right)\left(3 y_{0}-y_{\ell}\right)} \int d \tau\left\langle\ldots \phi_{\ell}(\tau)\right\rangle_{B_{\delta}}+\text { subleading in } \delta .
\end{gathered}
$$

Combining these two contributions gives the leading third order term.

At this point we repeat some important observations, also valid at higher orders in $\lambda$. 1) Only the almost marginal fields contribute, even in the intermediate stages. Other fields have $y$-values $\mathcal{O}(1)$ and so make subleading contributions. 2) Although this was on the disc, the result is largely independent of geometry.

One may repeat this analysis at each order in $\lambda$. Leading $\delta$ behaviour will come from the region of integration where all the perturbing fields come together simultaneously, can be evaluated using successive OPEs and will behave as $\lambda^{n} \delta^{1-n}$. The leading $\delta$ contribution of a general correlator is of the generic form,

$$
\begin{aligned}
\langle\ldots\rangle_{\lambda} & =\langle\ldots\rangle_{B_{\delta}}-\sum_{k \in M} \hat{\lambda}_{k} \int_{\partial \Sigma} d \tau\left\langle\ldots \phi_{k}(\tau)\right\rangle_{B_{\delta}}+\text { subleading in } \delta, \\
\hat{\lambda}_{0} & =\lambda-\lambda^{2} \frac{C_{00}{ }^{0}}{y_{0}}+\lambda^{3} \sum_{\ell \in M} \frac{C_{00}{ }^{\ell} C_{\ell 0}{ }^{0}+C_{00} C_{0 \ell}{ }^{0}}{2 y_{0}\left(2 y_{0}-y_{\ell}\right)}+\ldots, \\
\hat{\lambda}_{k} & =-\lambda^{2} \frac{C_{00}{ }^{k}}{2 y_{0}-y_{k}}+\lambda^{3} \sum_{\ell \in M} \frac{C_{00}{ }^{\ell} C_{\ell 0}{ }^{k}+C_{00}{ }^{\ell} C_{0 \ell}}{\left(2 y_{0}-y_{\ell}\right)\left(3 y_{0}-y_{k}\right)}+\ldots,
\end{aligned}
$$


however, the combinatorics required to find the exact answer to all orders would take many pages of exposition and is unnecessary in light of a short-cut using renormalisation group technology.

\subsection{RG calculation}

Note the answer (2.9) is already hinting at an RG connection, one interprets the $\hat{\lambda}_{k}$ as renormalised couplings replacing the bare coupling $\lambda$. A second clue is that at each order the leading $\delta$ contributions came from the region of integration where all the perturbing fields come together simultaneously. In a regulated correlator, this region is cut out and it's contribution absorbed into the renormalised couplings.

Consider a more general perturbation with an $\epsilon$-dependent regulator,

$$
S_{\mathrm{reg}}=\sum_{k \in F} \mu_{k}(\epsilon) \epsilon^{-y_{k}} \int_{\partial \Sigma} d \tau \phi_{k}(\tau)
$$

which leads to the following perturbative expansion for generic correlation functions $\langle\ldots\rangle_{\mu}$,

$$
\begin{aligned}
\langle\ldots\rangle_{\mu}=\left\langle\ldots e^{-S_{\mathrm{reg}}}\right\rangle_{B_{\delta}, \epsilon}=\langle\ldots\rangle_{B_{\delta}}-\sum_{k \in F} \mu_{k}(\epsilon) \epsilon^{-y_{k}} \int_{\partial \Sigma} d \tau\left\langle\ldots \phi_{k}(\tau)\right\rangle_{B_{\delta}} \\
\quad+\sum_{k, \ell \in F} \frac{\mu_{k}(\epsilon) \mu_{\ell}(\epsilon) \epsilon^{-y_{k}-y_{\ell}}}{2} \int_{\partial \Sigma} d \tau_{1} d \tau_{2} \rho_{\epsilon}\left(\tau_{1}, \tau_{2}\right)\left\langle\ldots \phi_{k}\left(\tau_{1}\right) \phi_{\ell}\left(\tau_{2}\right)\right\rangle_{B_{\delta}}+\ldots
\end{aligned}
$$

For the moment, the perturbation contains all boundary fields. $\rho$ denotes the regulator, we will use the usual step function $\rho_{\epsilon}\left(x_{1}, x_{2}\right)=\theta\left(\left|x_{1}-x_{2}\right|-\epsilon\right), \rho_{\epsilon}\left(x_{1}, x_{2}, x_{3}\right)=$ $\theta\left(\left|x_{1}-x_{2}\right|-\epsilon\right) \theta\left(\left|x_{2}-x_{3}\right|-\epsilon\right) \theta\left(\left|x_{3}-x_{1}\right|-\epsilon\right), \ldots$ Later we will show the result is independent of the choice of regulator. The coupling constants in the regulated correlators are defined such that the resulting correlator is independent of the (small) cut-off $\epsilon$. To second order in perturbation theory and using the step-function regulation we find the following renormalisation group equations,

$$
\epsilon \frac{d \mu_{k}(\epsilon)}{d \epsilon}=y_{k} \mu_{k}(\epsilon)-\sum_{i, j \in F} C_{i j}{ }^{k} \mu_{i}(\epsilon) \mu_{j}(\epsilon) .
$$

As $\epsilon \rightarrow 0$, the regulated correlator should reproduce the unregulated result,

$$
\lim _{\epsilon \rightarrow 0} \mu_{0}(\epsilon) \epsilon^{-y_{0}}=\lambda, \quad \lim _{\epsilon \rightarrow 0} \mu_{k}(\epsilon) \epsilon^{-y_{k}}=0, k \neq 0 .
$$

Together these equations fix the renormalised couplings and ensure that

$$
\langle\ldots\rangle_{\mu, \epsilon}=\langle\ldots\rangle_{\lambda} \text {. }
$$

To see what this has to do with the leading $\delta$ behaviour let us observe that equation (2.16) implies in particular that

$$
\begin{aligned}
& \sum_{k \in F} \mu_{k}(\epsilon) \epsilon^{-y_{k}} \int d \tau\left\langle\ldots \phi_{k}(\tau)\right\rangle \\
& \quad=\lambda \int d \tau\left\langle\ldots \phi_{0}(\tau)\right\rangle-\frac{\lambda^{2}}{2} \int d \tau_{1} d \tau_{2}\left(1-\rho_{\epsilon}\left(\tau_{1}, \tau_{2}\right)\right)\left\langle\ldots \phi_{0}\left(\tau_{1}\right) \phi_{0}\left(\tau_{2}\right)\right\rangle+\ldots,
\end{aligned}
$$


The renormalised couplings contain the contribution to the perturbed correlators cut-out by the cut-off. Using the OPE to write all the correlators in the same form we can equate coefficients,

$$
\begin{aligned}
\mu_{0}(\epsilon) \epsilon^{-y_{0}} & =\lambda-\frac{\lambda^{2}}{2} \int d u\left(1-\rho_{\epsilon}(\tau, \tau+u)\right) C_{00}{ }^{0}\left(2 \sin \frac{u}{2}\right)^{-1+y_{0}}+\ldots \\
& =\lambda-\lambda^{2} \frac{C_{00}{ }^{0} \epsilon^{y_{0}}}{y_{0}}+\ldots \\
\mu_{k}(\epsilon) \epsilon^{-y_{k}} & =-\frac{\lambda^{2}}{2} \int d u\left(1-\rho_{\epsilon}(\tau, \tau+u)\right) C_{00}{ }^{k}\left(2 \sin \frac{u}{2}\right)^{-1+2 y_{0}-y_{k}}+\ldots \\
& =-\lambda^{2} \frac{C_{00}{ }^{k} \epsilon^{2 y_{0}-y_{k}}}{2 y_{0}-y_{k}}+\ldots
\end{aligned}
$$

Since the leading behaviour comes from the removed area and the removed area is encoded in the renormalised couplings, all the leading behaviour must be encoded in the renormalised couplings. We can extract this by taking the leading behaviour of the renormalised couplings,

$$
\begin{aligned}
\mu_{0}(\epsilon) \epsilon^{-y_{0}} & =\lambda-\lambda^{2} \frac{C_{00} 0}{y_{0}}+\ldots, \\
\mu_{k}(\epsilon) \epsilon^{-y_{k}} & =-\lambda^{2} \frac{C_{00}{ }^{k}}{2 y_{0}-y_{k}}+\ldots .
\end{aligned}
$$

which we substitute back into (2.13). Noting that the renormalised couplings are $\mathcal{O}(\delta)$ and that the integrated regulated correlators are $\mathcal{O}(1)$ we reproduce the result of the brute force calculation,

$$
\langle\ldots\rangle_{\lambda}=\langle\ldots\rangle_{\mu, \epsilon}=\langle\ldots\rangle_{B_{\delta}}-\sum_{k \in F} \mu_{k}(\epsilon) \epsilon^{-y_{k}} \int_{\partial \Sigma} d \tau\left\langle\ldots \phi_{k}(\tau)\right\rangle_{A}+\text { subleading in } \delta
$$

wherein the renormalised couplings are given by (2.22)

We observe that there is a simplification that we can make. Since we are only interested in the leading $\delta \rightarrow 0$ behaviour of the renormalised couplings, looking at equations (2.14) and solving them as power series in $\lambda$ one can see this is encoded in the subset of equations involving only the almost marginal couplings, with all other couplings set to zero. This is the renormalisation group realisation of the fact that when we did the brute force calculation, we needed only the part of the OPEs involving the almost marginal fields.

Let us summarise our arguments. As seen from the brute force calculation, the leading $\delta$ behaviour of correlators came from the regions of integration where the all perturbing fields came together simultaneously. In a suitable renormalisation scheme, the contribution from these regions is cut-out and absorbed into the renormalised couplings. It should be emphasized that the expansion in powers of the renormalized coupling constants $\mu$ is simply a reorganization of the perturbative expansion in powers of $\lambda$, as is clear from equation (2.16). One can efficiently calculate these couplings by solving the RG equations with correct boundary conditions. The power of the method is illustrated by the fact that equations (2.19) and (2.21) can also be easily calculated from the renormalisation group equations (2.14). The leading $\delta$ behaviour of the solutions to the RG equations will then give the leading $\delta$ behaviour of the correlators via (2.23). 


\subsection{Toy Example : a single nearly marginal field}

As an illustration of our method, we consider the perturbation in a theory with a single nearly marginal field, $y=1-h=\delta^{2}$. We use $\delta^{2}$ to make comparison with the time-dependent formulation is easier.

$$
\langle\ldots\rangle_{\lambda}=\left\langle\ldots e^{-S_{\text {pert }}}\right\rangle_{B_{\delta}}, \quad S_{\text {pert }}=\lambda \int d \tau \phi(\tau) .
$$

In this case the RG equations are,

$$
\epsilon \frac{d \mu(\epsilon)}{d \epsilon}=\delta^{2} \mu(\epsilon)-C \mu(\epsilon)^{2}, \quad \lim _{\epsilon \rightarrow 0} \mu(\epsilon) \epsilon^{-\delta^{2}}=\lambda,
$$

which solve to give,

$$
\mu(\epsilon) \epsilon^{-\delta^{2}}=\frac{\delta^{2} \lambda}{\delta^{2}+C \epsilon^{\delta^{2}} \lambda}=\frac{\delta^{2} \lambda}{\delta^{2}+C \lambda}+\text { subleading in } \delta^{2},
$$

and hence the leading $\delta^{2}$ behaviour of the perturbed correlator is,

$$
\langle\ldots\rangle_{\lambda}=\langle\ldots\rangle_{B_{\delta}}-\frac{\delta^{2} \lambda}{\delta^{2}+C \lambda} \int d \tau\langle\ldots \phi(\tau)\rangle_{B_{\delta}}+\text { subleading in } \delta .
$$

In the present example it is possible to carry out the brute force calculation by expanding (2.24) in powers of $\lambda$, using the OPE to calculate the leading $\delta$ behaviour term by term and then resumming the series. The result coincides with (2.27).

Note that the series (2.24) has a finite radius of convergence. By performing the resummation we obtain a continuation to all values of $\lambda>0$ (for $\lambda<0$, the renormalised coupling becomes large and our perturbative assumptions break down). This is particularly important when one tries to calculate the perturbed correlation function at a nearby renormalization group fixed point. In this case the renormalized coupling constant stays small and approaches the fixed point value $\mu(\epsilon) \rightarrow \frac{\delta^{2}}{C}$ as $\lambda \rightarrow \infty$. The renormalized coupling constant captures information that is nonperturbative in $\lambda$.

\subsection{Scheme dependence}

The equations above have been derived using a step-function cut-off. In this section we consider a more general cut off $\rho_{\epsilon}\left(x_{1}, x_{2}\right)=\rho\left(\frac{1}{\epsilon}\left|x_{1}-x_{2}\right|\right)$. Re-deriving the RG equations we find,

$$
\epsilon \frac{d \mu_{k}(\epsilon)}{d \epsilon}=y_{k} \mu_{k}(\epsilon)-\sum_{i, j \in M} C_{i j}{ }^{k} \mu_{i}(\epsilon) \mu_{j}(\epsilon) f\left(y_{i}+y_{j}-y_{k}\right), \quad f(y)=\int_{0}^{\infty} d u u^{y} \frac{d \rho(u)}{d u} .
$$

What is important for our calculation is the leading $\delta$ behaviour, and one will note that since $\rho$ is a cut-off function (whose derivative is well behaved) we have

$$
\begin{aligned}
f(\delta) & =\rho(\infty)-\rho(0)+\text { subleading in } \delta \\
& =1+\text { subleading in } \delta .
\end{aligned}
$$


Hence as far as the leading $\delta$ behaviour is concerned, the result is scheme independent for all schemes whose cut-off cleanly removes all the behaviour from the integrals which would become divergent when $\delta \rightarrow 0$. Such schemes will subsequently be called "proper' 4 .

\section{Perturbative construction of time-dependent backgrounds}

The string backgrounds that we are interested in can be constructed as perturbations of the product conformal field theory $\mathrm{CFT}_{\mathrm{S}} \otimes \mathrm{CFT}_{\mathrm{X}_{0}}$. where $\mathrm{CFT}_{\mathrm{S}}$ is a conformal field theory with central charge $c=25$ representing the spatial part of the background and $\mathrm{CFT}_{\mathrm{X}_{0}}$ is a free boson CFT that is defined by the action

$$
S=-\frac{1}{4 \pi} \int d^{2} x \partial_{+} X_{0} \partial_{-} X_{0}
$$

The sign in front of the action means that $X_{0}$ is time-like.

We will be interested in certain perturbations of the static open string backgrounds which are characterized by a family of conformal boundary conditions $B_{\delta}$ for $\mathrm{CFT}_{\mathrm{S}}$ parametrized by a parameter $\delta$ together with Neumann boundary conditions for the $X_{0}$-CFT. The boundary state for the static background can be written as

$$
\left.\left|B_{\delta}\right\rangle\right\rangle_{\text {stat }} \equiv\left|B_{\delta}\right\rangle_{\mathrm{S}} \otimes|N\rangle_{\mathrm{X}_{0}}
$$

where $|N\rangle_{\mathrm{X}_{0}}$ is the boundary state associated to Neumann type boundary condition for the $X_{0^{-}}$ CFT. The boundary state of the time-dependent (dynamical) background that we are about to study will be denoted as $\left.\left|B_{\delta}\right\rangle\right\rangle_{\mathrm{dyn}}$. It may be formally constructed as follows,

$$
\left.\left|B_{\delta}\right\rangle\right\rangle_{\mathrm{dyn}} \equiv e^{-S_{\mathrm{Bd}}}\left|B_{\delta}\right\rangle_{\mathrm{S}} \otimes|N\rangle_{\mathrm{X}_{0}}
$$

where $S_{\mathrm{Bd}}$ is the following boundary action:

$$
S_{\mathrm{Bd}} \equiv \lambda \int_{\partial \Sigma} d x\left[e^{\delta X_{0}} \phi_{0}\right](x) .
$$

here we have fixed $y_{0}=\delta^{2}$ such that the perturbing field has conformal dimension $\delta^{2}+\left(1-\delta^{2}\right)=$ 1. There are cases where the short distance singularities in the OPE of $\left[e^{\delta X_{0}} \phi_{0}\right](x)$ with itself are integrable. It then follows that (3.32) indeed defines a conformal boundary state to all orders in a formal expansion in the parameter $\lambda$.

We observe an immediate problem: The perturbative expansion in $\lambda$ is not expected to be convergent since a shift of the zero mode of $X_{0}$ is equivalent to a rescaling of $\lambda$. In the following subsection we will describe how this problem may be solved by fixing the zero mode value. We will then explain how to calculate the boundary state $\left|B_{\delta}\right\rangle_{\text {dyn }}$ to leading order in $\delta$ by generalizing the RG techniques from the previous section to the time-dependent case.

In order to explain our method we will assume that the short distance singularities in the OPE of $\left[e^{\delta X_{0}} \phi_{0}\right](x)$ with itself are integrable. This assumption is made for pedagogical purposes only, our main conclusions do not depend on it, as is shown in Appendix A.

\footnotetext{
${ }^{4}$ Using the equations like those derived in this section one could easily give some sufficient conditions for the definition of such a scheme.
} 


\subsection{The $X_{0}$ BCFT}

To begin with, let us consider the $X_{0}$-CFT on the cylinder with periodic boundary conditions in space direction $\sigma$. The space of states of the $X_{0}$ theory is generated from a continuum of states $\left\langle\left.\omega\right|_{X_{0}}, \omega \in \mathbb{R}\right.$ satisfying

$$
\left\langle\left.\omega\right|_{\mathrm{X}_{0}} L_{-n}=0=\left\langle\left.\omega\right|_{\mathrm{X}_{0}} \bar{L}_{-n}, \quad n>0, \quad\left\langle\left.\omega\right|_{\mathrm{X}_{0}} L_{0}=-\omega^{2}\left\langle\left.\omega\right|_{\mathrm{X}_{0}}\right.\right.\right.\right.
$$

where $L_{n}, \bar{L}_{n}$ are the generators of the $c=1$ Virasoro algebra.

In order to characterize the perturbed boundary state $\left.\left|B_{\delta}\right\rangle\right\rangle_{\mathrm{dyn}}$ one would naturally consider the amplitude

$$
A(a, \omega) \equiv\left\langle\left\langle a, \omega \mid B_{\delta}\right\rangle_{\mathrm{dyn}}, \quad\left\langle\langle a , \omega | \equiv \left\langlea | _ { \mathrm { S } } \otimes \left\langle\left.\omega\right|_{\mathrm{x}_{0}}\right.\right.\right.\right.
$$

where $\left\langle\left. a\right|_{\mathrm{S}}\right.$ is a highest weight state in the $\mathrm{CFT}_{\mathrm{S}}$ on the cylinder. However, as indicated in the introduction to this section one can not expect that the perturbative expansion in powers of $\lambda$ will be useful to determine $A(a, \omega)$.

In order to overcome this problem, let us introduce the zero mode $x_{0}=\int_{0}^{2 \pi} d \sigma X_{0}(\sigma, 0)$. We may fix the zero mode $x_{0}$ by considering amplitudes which involve the eigenstates $\left\langle\left. t\right|_{\mathrm{X}_{0}}\right.$ of $x_{0}$. The states $\left\langle\left. t\right|_{\mathrm{X}_{0}}\right.$ are obtained from $\left\langle\left.\omega\right|_{\mathrm{X}_{0}}\right.$ by Fourier-transformation,

$$
\left\langle\left. t\right|_{\mathrm{X}_{0}}=\int_{\mathbb{R}} d \omega e^{i \omega t}\left\langle\left.\omega\right|_{\mathrm{X}_{0}} .\right.\right.
$$

It follows that the states $\left\langle\left. t\right|_{\mathrm{X}_{0}}\right.$ satisfy

$$
\left\langle\left. t\right|_{\mathrm{X}_{0}} L_{-n}=0=\left\langle\left. t\right|_{\mathrm{X}_{0}} \bar{L}_{-n}, \quad n>0, \quad\left\langle\left. t\right|_{\mathrm{X}_{0}} x_{0}=t\left\langle\left. t\right|_{\mathrm{X}_{0}} .\right.\right.\right.\right.
$$

Instead of considering $A(a, \omega)$, we will first determine the amplitude

$$
A(a, t) \equiv\left\langle\left\langle a, t \mid B_{\delta}\right\rangle\right\rangle_{\mathrm{dyn}}, \quad\left\langle\langle a , t | \equiv \left\langlea | _ { \mathrm { S } } \otimes \left\langle\left. t\right|_{\mathrm{X}_{0}} .\right.\right.\right.
$$

We will interpret $A(a, t)$ as an amplitude which directly represents the time evolution of the boundary state $\left.\left|B_{\delta}\right\rangle\right\rangle_{\text {dyn }}$.

The perturbative expansion for $A(a, t)$ in powers of $\lambda$ involves amplitudes like

$$
\left\langle t\left|: e^{\delta X_{0}\left(\sigma_{1}\right)}: \ldots: e^{\delta X_{0}\left(\sigma_{n}\right)}:\right| N\right\rangle_{X_{0}}=e^{n \delta t} \prod_{r<s}\left|2 \sin \frac{\sigma_{r}-\sigma_{s}}{2}\right|^{2 \delta^{2}} .
$$

The prefactor $e^{n \delta t}$ comes from the zero mode dependence of the normal ordered exponentials $e^{\delta X_{0}(\sigma)}$ together with (3.37). The $\sigma_{r}$-dependent factors follow from the OPE

$$
: e^{\delta X_{0}\left(\sigma_{2}\right)}:: e^{\delta X_{0}\left(\sigma_{1}\right)}:=\left|2 \sin \frac{\sigma_{2}-\sigma_{1}}{2}\right|^{2 \delta^{2}}: e^{\delta X_{0}\left(\sigma_{2}\right)} e^{\delta X_{0}\left(\sigma_{1}\right)}:,
$$

which is the usual OPE of normal ordered exponentials of a free field up to the change of sign in the exponent of the short-distance factor due to the minus sign in front of the kinetic term for $X_{0}$. 
The time-like nature of the $X_{0}$-CFT is directly responsible for the fact that the short-distance behavior of the operator product $e^{\delta X_{0}\left(\sigma_{2}\right)} e^{\delta X_{0}\left(\sigma_{1}\right)}$ is nonsingular. It follows that

$$
\left\langle t\left|: e^{\delta X_{0}\left(\sigma_{1}\right)}: \ldots: e^{\delta X_{0}\left(\sigma_{n}\right)}:\right| N\right\rangle_{X_{0}}=e^{n \delta t}\left(1+\mathcal{O}\left(\delta^{2}\right)\right)
$$

holds as long as $n$ is not of the order $\delta^{-1}$.

With these definitions one may use equations (3.32) and (3.38) to generate a formal series expansion of $A(a, t)$ in powers of $\lambda e^{\delta t}$. We expect that the radius of convergence of this expansion is finite, as will be confirmed below. We will nevertheless be able to find explicit representations for $\left.\left|B_{\delta}\right\rangle\right\rangle_{\text {dyn }}$ and $A(a, t)$ which are valid to leading order in $\delta$ but for arbitrary $\lambda e^{\delta t}$ by using the renormalization group resummation of the naive perturbative expansion in powers of $\lambda$ as discussed in Section 2 .

\subsection{RG improvement in the time-dependent case}

To represent the decay process in string theory, we couple our CFT to a time-like boson with a Neumann-type boundary condition. We then perturb the theory by the (truly) marginal operator,

$$
S=\lambda \int d x\left[e^{\delta X^{0}} \phi_{0}\right](x)
$$

with $y_{0}=\delta^{2}$. Using the technology above, this leads us to consider the regulated perturbation,

$$
S_{\text {reg }}=\sum_{k \in M} \sum_{n=1}^{\infty} u_{k, n} \epsilon^{n^{2} \delta^{2}-y_{k}} \int d x\left[e^{n \delta X^{0}} \phi_{k}\right](x) .
$$

One may wonder why we did not expand $U\left(X_{0}\right)$ and $\lambda_{P}\left(X_{0}\right)$ into "Fourier modes" : $e^{-i \omega X_{0}}$ :, as has been done e.g. in [FHL]. In this case one would find fields in the boundary action with arbitrarily negative conformal dimensions, which would in particular create problems in the application of RG techniques. We have included all the near marginal boundary fields which are generated in the OPE of the perturbing field $\left[e^{\delta X_{0}} \phi_{0}\right](x)$ with itself.

The conditions for the $\epsilon$-independence of the correlation functions are then found to be

$$
\begin{gathered}
\epsilon \frac{d u_{k, n}}{d \epsilon}=\left(y_{k}-n^{2} \delta^{2}\right) u_{k, n}-\sum_{i, j \in M} \sum_{m=1}^{n-1} C_{i j}^{k} u_{i, m} u_{j, n-m}, \\
\lim _{\epsilon \rightarrow 0} u_{0,1}=\lambda, \quad \lim _{\epsilon \rightarrow 0} u_{k, n} \epsilon^{n^{2} \delta^{2}-y_{k}}=0 .
\end{gathered}
$$

These equations can be solved recursively,

$$
u_{0,1}=\lambda, \quad u_{k, 1}=0, \quad u_{k, n}=\frac{1}{y_{k}-n^{2} \delta^{2}} \sum_{i, j \in M} \sum_{m=1}^{n-1} C_{i j}{ }^{k} u_{i, m} u_{j, n-m} .
$$

This is also a fixed point of (3.43), as was to be expected because our original perturbation was truly marginal. The equations above can be translated into a system of evolution equations by 
introducing

$$
U_{k}(t)=\sum_{n=1}^{\infty} u_{k, n} e^{n \delta t}
$$

It easily follows that $U_{k}(t)$ is a solution of the equations

$$
\ddot{U}_{k}(t)=y_{k} U_{k}(t)-\sum_{i, j \in M} C_{i j}{ }^{k} U_{i}(t) U_{j}(t)
$$

supplemented by the boundary conditions

$$
U_{0}(t)=\lambda e^{\delta t}+\mathcal{O}\left(e^{2 \delta t}\right), \quad U_{k}(t)=\mathcal{O}\left(e^{2 \delta t}\right), \quad k \neq 0
$$

When we compare this system with the renormalization group equations (2.14) of the timeindependent treatment, we clearly would not expect any simple relation between the solutions to the respective equations. We will see an explicit example in a moment.

The leading behaviour for $\delta \rightarrow 0$ of time dependent correlators $\langle\ldots\rangle_{\lambda}$ is then given as

$$
\langle\ldots\rangle_{\lambda}=\langle\ldots\rangle_{B_{\delta} \otimes N}-\sum_{k \in F} \sum_{n=1}^{\infty} u_{k, n} \epsilon^{n^{2} \delta^{2}-y_{k}} \int d \tau\left\langle\ldots\left[e^{n \delta X_{0}} \phi_{k}\right](\tau)\right\rangle_{B_{\delta} \otimes N} .
$$

Of particular interest is the amplitude $A(a, t)$ defined in equation (3.38) above. The leading behavior of this quantity may be represented as

$$
\begin{aligned}
A(a, t) \underset{\delta \rightarrow 0}{\sim}\left\langle\left\langle a, t \mid B_{\delta}\right\rangle_{\text {stat }}-\sum_{k \in F} \sum_{n=1}^{\infty} u_{k, n} \epsilon^{n^{2} \delta^{2}-y_{k}} \int d \tau\left\langle\left\langle a, t\left|\left[e^{n \delta X_{0}} \phi_{k}\right](\tau)\right| B_{\delta}\right\rangle\right\rangle_{\text {stat }}\right. \\
\underset{\delta \rightarrow 0}{\sim}\left\langle a \mid B_{\delta}\right\rangle_{\mathrm{S}}-\sum_{k \in F} U_{k}(t) \int d \tau\left\langle a\left|\phi_{k}(\tau)\right| B_{\delta}\right\rangle_{\mathrm{S}} .
\end{aligned}
$$

We observe that to leading order in $\delta$ one may represent the time-dependence of the perturbed amplitudes $A(a, t)$ rather simply in terms of the solutions to the evolution equations (3.47).

A few comments are at order at this point. A priori we may expect the representation (3.50) to be useful only for times $t$ which are sufficiently small to ensure the convergence of the series (3.46). The possibility to find representations for the amplitude $A(a, t)$ valid for all times $t$ depends crucially on whether the dynamics defined by the equations (3.47), (3.48) will remain bounded or not. If not, one would violate the condition that $U_{k}=\mathcal{O}(\delta)$ after some time. The possibility of unbounded motions is raised by the fact that the right hand side of (3.47) is the force from a cubic potential which is unbounded from below. We will not be able to offer a general answer to this question, but we will prove boundedness of the dynamics defined by (3.47), (3.48) in two interesting cases below.

If the functions $U_{k}(t)$ have a well defined limit as $t \rightarrow \infty$ it necessarily has to be a fixed point of the time-independent RG flow. However, without more information, there is no reason to expect this fixed point will be the same as the the end point of the RG flow that is generated by 
the boundary field $\phi_{0}$. We will indeed illustrate in the next subsection that this is not the case in general.

Let us finally remark that both the evolution equations and the RG equation for the time independent system are scheme dependent in general. Furthermore, there is no reason to suppose that a scheme chosen for one system should be related to a scheme chosen for the other in a simple way. However, as argued in section 2.4 the leading behaviour of both systems for $\delta \rightarrow 0$ is scheme independent.

\subsection{Toy Example : a single marginal field}

We continue our example from section 2.3. To create the time-dependent version of this system, we couple our single near marginal field to the time-like boson,

$$
S=\lambda \int d x\left[e^{\delta X^{0}} \phi\right](x)
$$

Using the technology of RG, this leads us to consider the more general perturbation,

$$
S_{\mathrm{reg}}=\sum_{n=1}^{\infty} u_{n} \epsilon^{\left(n^{2}-1\right) \delta^{2}} \int d x\left[e^{n \delta X^{0}} \phi\right](x)
$$

and the RG equations,

$$
\epsilon \frac{d u_{n}}{d \epsilon}=\left(1-n^{2}\right) \delta^{2} u_{n}-\sum_{m=1}^{n-1} C u_{m} u_{n-m}, \quad \lim _{\epsilon \rightarrow 0} u_{1}=\lambda, \quad \lim _{\epsilon \rightarrow 0} u_{n} \epsilon^{n^{2} \delta^{2}-y_{k}}=0 .
$$

Which have the solution

$$
u_{n}=(-1)^{n-1} n \lambda\left(\frac{C \lambda}{6 \delta^{2}}\right)^{n-1}
$$

To understand the solution better we consider the combination

$$
U(t)=\sum_{n=1}^{\infty} u_{n} e^{n \delta t}=\frac{3 y}{2 C} \frac{1}{\cosh ^{2} \delta\left(t-t_{0}\right)}, \quad e^{\delta t_{0}}=\frac{6 y \lambda}{C} .
$$

Which satisfies

$$
\ddot{U}(t)=\delta^{2} U(t)-C U(t)^{2}
$$

This is the equation of motion for a particle in a cubic potential. The solution above represents the particle leaving $U=0$ in the infinite past, falling toward the minimum $U_{*}=\frac{\delta^{2}}{C}$, moving on up the other side before coming to instantaneous rest at a point $U\left(t_{0}\right)=\frac{3}{2} U_{*}$. The particle then returns to $U=0$ in the infinite future.

For systems with only a finite number of nearly marginal fields, such oscillatory behaviour will be generic. To find a system of a dissipative nature, we need an infinite number of fields, as is found in the example that we will consider next. 


\section{4. (Perturbed) Liouville BCFT}

Liouville theory on the upper half plane $\mathbb{U}$ is defined semiclassically by means of the action

$$
S_{\mathrm{L}}[\phi] \equiv \frac{1}{\pi} \int_{\mathbb{U}} d^{2} z\left(\partial \phi \bar{\partial} \phi+\pi \mu e^{2 b \phi}\right)+\mu_{B} \int_{\mathbb{R}} d x e^{b \phi} .
$$

The corresponding boundary conditions for the Liouville field are of Neumann-type,

$$
i(\partial-\bar{\partial}) \phi=2 \pi b \mu_{\mathrm{B}} e^{b \phi} .
$$

One of the interesting implications of the exact solution of boundary Liouville theory [FZZ, TT1, Ho, PT, T2] is the fact that the boundary conditions of the corresponding quantum theory are not uniquely parametrized by the parameter $\mu_{\mathrm{B}}$ which appears in the classical case (4.2). For each value of $\mu_{\mathrm{B}}$ there are countably many different boundary conditions which have quite different physical properties. In the following we will elaborate on the string theoretical consequences of this phenomenon, extending the previous discussion in [T2].

We will exclusively consider the case $b=1$ in this paper, which corresponds to central charge $c_{\mathrm{L}}=25$. The primary fields of the theory are distinguished by their conformal weights, $\Delta$, and will be denoted by $V_{\alpha}(\tau, \sigma)$ in the bulk and $\Phi_{P}(x)$ on the boundary. The labels $P$ and $\alpha$ will be used interchangably and are related to each other and the conformal weight via $\Delta_{\alpha}=1+P^{2}=\alpha(2-\alpha), \alpha=1+i P$.

\section{Boundary state}

The boundary states which correspond to the classical boundary condition (4.2) were first presented in [FZZ]. They can be represented as Ishibashi-states $|P\rangle_{\text {Ish }}$,

$$
\left|B_{\delta}\right\rangle_{\mathrm{L}}=\int_{0}^{\infty} \frac{d P}{2 \pi} A_{P}^{\delta}|P\rangle_{\text {Ish }}
$$

where $|P\rangle_{\text {Ish }}$ is the Ishibashi state built upon the bulk Liouville heighest weight state $|P\rangle$ corresponding to the vertex operator $V_{\alpha}$. The one-point function $A_{P}^{\delta}$ depends on a parameter $\delta$ which is related to the boundary cosmological constant $\mu_{\mathrm{B}}$ via

$$
\cos \pi(1+\delta)=\frac{\mu_{\mathrm{B}}}{\sqrt{\mu}} .
$$

The explicit expression for the coefficients $A_{P}^{\delta}$ is then given as

$$
A_{P}^{\delta}=\frac{\cosh (2 \pi P(1+\delta))}{2(\sinh 2 \pi P)^{2}} \Theta(P), \quad \Theta(P):=\frac{4 \pi i P \mu^{i P}}{(\Gamma(1+2 i P))^{2}} .
$$

It has turned out to be useful to split off the function $\Theta(P)$ as a normalizing factor.

We will mainly be interested in the case of small values of $\delta$, which corresponds to the region around the first minimum of $\mu_{\mathrm{B}}=\mu_{\mathrm{B}}(\delta)$ on the real positive half-axis. Bear in mind that $-\delta$ corresponds to the same value of $\mu_{\mathrm{B}}$.

\footnotetext{
${ }^{5}$ Other parametrizations have been used in the literature. $\delta$ is related to the parameter $\sigma$ from [PT, [T2] as $2 \sigma=1-\delta$, whereas the parameter $s$ from [FZZ] is related to $\delta$ as $s=i(1+\delta)$.
} 


\subsection{Hamiltonian picture - closed string channel}

We would like to understand the qualitative differences between the cases $\delta>0$ and $\delta<$ 0 . Some useful insight can be obtained by considering the Hamiltonian picture for Liouville theory which is naturally associated to the world-sheet being the cylinder. The boundary state is considered as description for the initial state at $\tau=0$. We may consider expectation values like

$$
\left\langle 0\left|V_{\alpha_{n}}\left(\tau_{n}, \sigma_{n}\right) \ldots V_{\alpha_{1}}\left(\tau_{1}, \sigma_{1}\right)\right| B_{\delta}\right\rangle_{\mathrm{L}}
$$

It is natural to interpret the zero mode $\phi_{0} \equiv \int_{0}^{2 \pi} d \sigma \phi(\sigma)$ as a coordinate for the target space of Liouville theory. In order to discuss localization properties in the target space it is useful to think about the states in $\mathcal{H}_{\mathrm{L}}$ in terms of the Schrödinger representation 6 for the zero mode $\phi_{0}$. States $|\Psi\rangle_{\mathrm{L}}$ are then represented by wave-functions $\Psi\left(\phi_{0}\right) \in \mathcal{H}\left(\phi_{0}\right)$, and the norm $\|\Psi\|^{2}$ is represented in the form

$$
\|\Psi\|^{2} \equiv \int d \phi_{0}\left\|\Psi\left(\phi_{0}\right)\right\|_{\mathcal{H}\left(\phi_{0}\right)}^{2}
$$

The norm density $\left\|B_{\delta}\left(\phi_{0}\right)\right\|_{\mathcal{H}\left(\phi_{0}\right)}^{2}$ of the wave-function associated to the boundary state $\left|B_{\delta}\right\rangle_{\mathrm{L}}$ can then be seen as describing the "profile" of the D-branes associated to the boundary condition with label $\delta$. At present we do not know how to calculate these profiles explicitly, but the asymptotic behavior can be read off from the asymptotic behavior of the one-point function for $P \rightarrow 0$ and $P \rightarrow \infty$ respectively. In order to see this let us consider the representation of $A_{P}^{\delta}$ as an overlap,

$$
A_{P}^{\delta}=\left\langle P \mid B_{\delta}\right\rangle_{\mathrm{L}}=\int d \phi_{0}\left\langle\Psi_{P}\left(\phi_{0}\right) \mid B_{\delta}\left(\phi_{0}\right)\right\rangle_{\mathcal{H}\left(\phi_{0}\right)}
$$

Let us first consider the asymptotics $\phi_{0} \rightarrow-\infty$. One should keep in mind that the wavefunction $\Psi_{P}\left(\phi_{0}\right)$ behaves as [ $\left.\underline{\mathrm{ZZ}}, \underline{\mathrm{T} 0}\right]$

$$
\Psi_{P}\left(\phi_{0}\right) \underset{\phi_{0} \rightarrow \infty}{\sim}\left(e^{2 i P \phi_{0}}+R(P) e^{-2 i P \phi_{0}}\right) \Omega
$$

where $\Omega$ is the Fock vacuum, and $R(P)$ is the reflection amplitude. The divergence found in the wave-function of the boundary state when $P \rightarrow 0$,

$$
\left|A_{P}^{\delta}\right| \underset{P \rightarrow 0}{\sim} \frac{1}{2 \pi P}
$$

is most naturally explained if $B_{\delta}\left(\phi_{0}\right)$ approaches a constant for $\phi_{0} \rightarrow-\infty$.

In order to discuss the asymptotics of $B_{\delta}\left(\phi_{0}\right)$ for $\phi_{0} \rightarrow+\infty$ let observe that these asymptotics are related to the asymptotics for $P \rightarrow \infty$ of $A_{P}^{\delta}$. Indeed, for large $P$ one may expect that the rapid oscillations of the wave function $\Psi_{P}\left(\phi_{0}\right)$ will average out the contributions to the integral (4.8) from a large range of values of $\phi_{0}$. This range is roughly bounded from above by the turning point of the motion of a string in the purely repulsive Liouville potential. The

\footnotetext{
${ }^{6}$ The zero mode $\phi_{0}$ is an operator which can be constructed from the exponential fields of Liouville theory [T0]. It is unbounded, but well-defined on a dense domain, symmetric, $\phi_{0}^{\dagger}=\phi_{0}$, and it seems likely that $\phi_{0}$ has a self-adjoint extension.
} 
purely repulsive nature of the potential furthermore implies that $\Psi_{P}\left(\phi_{0}\right)$ will decay rapidly for $\phi_{0} \rightarrow+\infty$. It follows that the main contributions to the integral (4.8) come from the region around the turning point of the motion of a string in the Liouville potential. The latter will grow with $P$. The asymptotics of $\left|A_{P}^{\delta}\right|$ for large $P$

$$
\left|A_{P}^{\delta}\right|=\frac{\sinh (2 \pi(1+\delta) P)}{\sinh (2 \pi P)} \underset{P \rightarrow \infty}{\sim} e^{2 \pi \delta P},
$$

therefore reflects the asymptotics of the wave-function $B_{\delta}\left(\phi_{0}\right)$ for $\phi_{0} \rightarrow \infty$. Note in particular that the latter depends decisively on the sign of $\delta$.

The resulting picture looks as follows: For each value of $\mu_{\mathrm{B}}$ we find two different boundary states with $-1<\delta<1$ distinguished by the value of $\operatorname{sgn}(\delta)$, one of which $(\operatorname{sgn}(\delta)>0)$ has a strong growth of the profile $M\left(\phi_{0}\right) \equiv\left\|B_{\delta}\left(\phi_{0}\right)\right\|^{2}$ for $\phi_{0} \rightarrow \infty$. In string theoretic terms one may interpret this fact as the existence of a concentration of "mass" (in the sense of source for closed strings) for large values of $\phi_{0}$ on those D1 branes which have $\delta>0$. An intuitive way to visualize the profiles for the two cases $\delta<0$ and $\delta>0$ is given in figures 1 and 2 respectively.

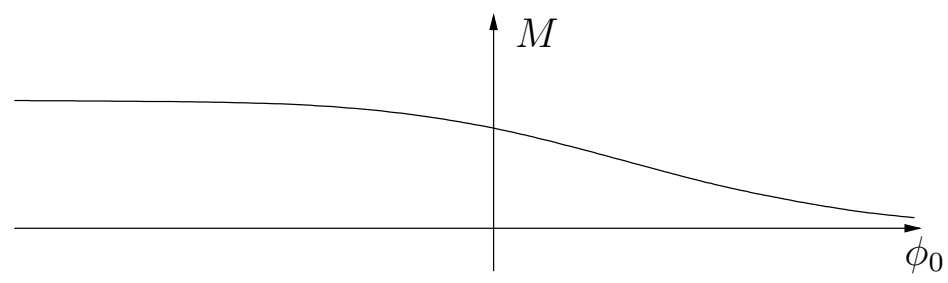

Figure 1: Qualitative visualization of the D1 brane profile $M\left(\phi_{0}\right)$ for $\delta<0$.

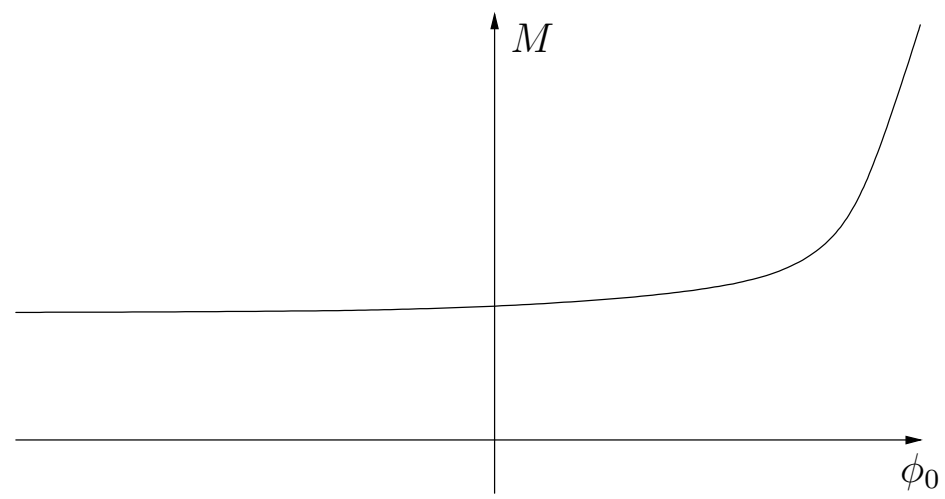

Figure 2: Qualitative visualization of the D1 brane profile $M\left(\phi_{0}\right)$ for $\delta>0$.

\subsection{Boundary fields}

It is sometimes useful to observe (see appendix $\mathbb{B}$ for more details) that fields $\Phi_{\alpha}^{\delta}(\sigma)$ localized on the boundary can be defined with the help of

$$
\Phi_{P}^{\delta}(\sigma)\left|B_{\delta}\right\rangle_{\mathrm{L}} \equiv C_{\delta}(P) \lim _{\tau \rightarrow 0}(2 \tau)^{2 \Delta_{\alpha}-\Delta_{2 \alpha}} V_{\alpha}(\tau, \sigma)\left|B_{\delta}\right\rangle_{\mathrm{L}}
$$


where $P$ and $\alpha$ are related by $\alpha=1+i P . C_{\delta}(P)$ is a certain normalizing factor defined in appendix A that we do not need explicitly except at the point $C_{\delta}(i \delta)=0$. It has been chosen in such a way that the boundary fields $\Phi_{P}^{\delta}(\sigma)$ are symmetric under $P \rightarrow-P, \Phi_{P}^{\delta}(\sigma)=\Phi_{-P}^{\delta}(\sigma)$. It will later be important for us to observe that the asymptotics of $\Phi_{i \varpi}^{\delta}(x)$ for $\phi_{0} \rightarrow-\infty$ can be represented as follows

$$
\Phi_{i \varpi}^{\delta}(x) \underset{\phi_{0} \rightarrow-\infty}{\sim} e^{\phi_{0}}\left(C_{\delta}(i \varpi): e^{-\varpi \phi}:+C_{\delta}(-i \varpi): e^{+\varpi \phi}:\right)
$$

The first term is directly understood from $V_{\alpha}(\tau, \sigma) \sim: e^{2 \alpha \phi}(\tau, \sigma):$, the second is found taking into account that $\Phi_{P}^{\delta}(\sigma)=\Phi_{-P}^{\delta}(\sigma)$.

In order to characterize the boundary fields $\Phi_{P}^{\delta}(x)$ completely we need to know both the operator product expansions (OPEs) and the three point functions of these fields. The OPE is known [T2] to be of the form

$$
\begin{aligned}
\Phi_{P_{2}}^{\delta}\left(x_{2}\right) \Phi_{P_{1}}^{\delta}\left(x_{1}\right)=\int_{0}^{\infty} d P_{3} & F_{P_{2} P_{1}}^{P_{3}}\left|x_{2}-x_{1}\right|^{\Delta_{P_{3}}-\Delta_{P_{2}}-\Delta_{P_{1}}} \Phi_{P_{3}}^{\delta}\left(x_{1}\right) \\
& +f_{P_{2} P_{1}}^{\vartheta}\left|x_{2}-x_{1}\right|^{\Delta_{\vartheta}-\Delta_{P_{2}}-\Delta_{P_{1}}} \Phi_{\vartheta}^{\delta}\left(x_{1}\right)+\text { descendants . }
\end{aligned}
$$

Of particular importance for us is the term in the second line of (4.14) which is proportional to the field $\Phi_{\vartheta}^{\delta}\left(x_{1}\right)$, with $\vartheta$ being defined as

$$
\vartheta=i \delta .
$$

The field $\Phi_{\vartheta}^{\delta}\left(x_{1}\right)$ has conformal dimension $\Delta_{\vartheta}=1-\delta^{2}<1$. It will therefore correspond to an open string tachyon in the $c=1$ noncritical string theory that we are about to study. The fact that $\Phi_{\vartheta}^{\delta}\left(x_{1}\right)$ appears discretely in (4.14) indicates that it creates a bound state in the spectrum of boundary Liouville theory on the strip, as will be further discussed in subsection 4.3 below. The OPE coefficient $f_{P_{2} P_{1}}^{\vartheta}$ is nonvanishing only if $\delta>0$.

We will need the explicit formula for the OPE coefficients $F_{P_{2} P_{1}}^{P_{3}}$ only in the case when $\delta \rightarrow 0$ with $P_{k}=\mathcal{O}(\delta), k=1,2,3$,

$$
F_{P_{2} P_{1}}^{P_{3}} \underset{\delta \rightarrow 0}{\sim} \frac{1}{2 \pi} \frac{4 P_{3}^{2}}{\delta^{2}+P_{3}^{2}} .
$$

This formula is proven in the Appendix B, As noted above, we have $f_{P_{2} P_{1}}^{\vartheta}=0$ for $\delta<0$. The OPE is then defined for $\delta>0$ by analytic continuation with respect to the parameter $\delta$, see [T2] for more details. One picks up extra contributions from poles of $F_{P_{2} P_{1}}^{P_{3}}$ which cross the contour of integration in (4.14). It then follows easily from (4.16)

$$
f_{P_{2} P_{1}}^{\vartheta}=-2 \pi i \underset{P_{3}=i \delta}{\operatorname{Res}} F_{P_{2} P_{1}}^{P_{3}} \underset{\delta \rightarrow 0}{\sim} 2 \delta .
$$

Note that the formulae (4.16) and (4.17) also cover the cases when $P_{2}$ or $P_{1}$ take the value $\vartheta$.

We will also need the bulk-boundary structure function $\left\langle P_{1}\left|\Phi_{P_{2}}^{\delta}(1)\right| B_{\delta}\right\rangle_{\mathrm{L}}$. It is shown in Appendix $\mathrm{B}$ that in the limit $\delta \rightarrow 0, P_{2}=\mathcal{O}(\delta)$ we find the following behavior

$$
\left\langle P_{\mathbf{1}}\left|\Phi_{P_{2}}^{\delta}(1)\right| B_{\delta}\right\rangle_{\mathrm{L}} \underset{\delta \rightarrow 0}{\sim} \frac{1}{2 \pi} \frac{2 \pi P_{\mathbf{1}}}{\sinh 2 \pi P_{\mathbf{1}}} \Theta\left(P_{\mathbf{1}}\right),
$$

which is independent of $P_{2}$. 


\subsection{Hamiltonian picture - open string channel}

There is an alternative Hamiltonian representation which is associated to the world-sheet being the strip $\mathbb{S}$. The corresponding Hilbert space of the boundary Liouville theory with boundary condition parametrized by $\delta$ on both sides of the strip may then be represented as follows [T1, T2]

$$
\mathcal{H}_{\delta \delta}^{\mathrm{B}}=\int_{\mathbb{R}_{+}}^{\oplus} d P \mathcal{V}_{P} \oplus\left\{\begin{array}{r}
\emptyset \text { for } \delta<0, \\
\mathcal{V}_{\vartheta} \text { for } \delta>0,
\end{array}\right.
$$

where $\mathcal{V}_{P}$ is the irreducible unitary representation of the Virasoro algebra with $c=25$ which has highest weight $\Delta_{P}=1+P^{2}$. State-operator correspondence therefore yields the usual relation between the OPE (4.14) of boundary fields to a summation over a basis for the Hilbert space $\mathcal{H}_{\delta \delta}^{\mathrm{B}}$.

We want to show that the additional contributions which occur in the spectrum 4.19) can be interpreted as bound states. This can be seen more clearly by representing the states in terms of the Schrödinger representation for the zero mode $\left.\phi_{0}^{\mathrm{op}} \equiv \int_{0}^{\pi} d \sigma \phi(\sigma, \tau)\right|_{\tau=0}$. which is naturally associated to the given Hamiltonian picture. The eigenstates of the Hamitonian $\mathbf{H}$ are then represented by wave-functions $\Psi_{P}\left(\phi_{0}^{\mathrm{op}}\right)$ which have an asymptotic behavior for $\phi_{0}^{\mathrm{op}} \rightarrow-\infty$ of the following form

$$
\Psi_{P}\left(\phi_{0}^{\mathrm{op}}\right) \underset{\phi_{0}^{\mathrm{op}} \rightarrow-\infty}{\sim}\left(C_{\delta}(P) e^{i P \phi_{0}^{\mathrm{op}}}+C_{\delta}(-P) e^{-i P \phi_{0}^{\mathrm{op}}}\right) \Omega
$$

where $\Omega$ is the Fock vacuum. Our previous observation that $C_{\delta}(\vartheta)=0$ therefore implies the exponential decay of $\Psi_{\vartheta}\left(\phi_{0}^{\mathrm{op}}\right)$ for $\phi_{0}^{\mathrm{op}} \rightarrow \infty$ characteristic for a bound state.

In the context of the $c=1$ noncritical string theory there will be a single physical state $|\vartheta\rangle\rangle$ which is constructed by tensoring the highest weight state in $\mathcal{V}_{\vartheta}$ with a suitable "dressing" from the CFT associated to the time direction $X_{0}$. From the string theoretical point of view it therefore seems natural to interpret the concentration of "mass" as depicted in figure 2 as a potential sink for the open strings on the D1 branes. The attractive force associated with the potential sink may bind open strings.

\subsection{Perturbed boundary Liouville theory}

We will consider the perturbation of boundary Liouville theory which corresponds to the boundary action

$$
S_{\mathrm{Bd}}=\lambda \int_{\partial \Sigma} d x \Phi_{\vartheta}^{\delta}(x)
$$

The perturbed boundary state is then formally defined as

$$
\left|B_{\delta}\right\rangle_{\mathrm{L}}^{\text {pert }}=e^{-S_{\mathrm{Bd}}}\left|B_{\delta}\right\rangle_{\mathrm{L}} .
$$

Our aim is to extract the leading behavior of the perturbed boundary state $\left|B_{\delta}\right\rangle_{\mathrm{L}}^{\text {pert }}$ for $\delta \rightarrow 0$. As discussed in section 2 we may use the renormalization group to resum the relevant contributions of the perturbative expansion in powers of $\lambda$ into renormalized couplings. 


\section{RG flow equations}

In order to apply the discussion from section 2 to the case at hand let us introduce a proper regularization scheme with cut-off $\epsilon$ and consider the boundary action

$$
S_{\mathrm{Bd}}^{\mathrm{ren}}=\int_{\partial \Sigma} d x\left(u \epsilon^{-\delta^{2}} \Phi_{\vartheta}^{\delta}(x)+\int_{0}^{\infty} d P \lambda(P) \epsilon^{P^{2}} \Phi_{P}^{\delta}(x)\right)
$$

which contains the renormalized coupling constants $u \equiv u_{\epsilon}$ and $\lambda(P) \equiv \lambda_{\epsilon}(P)$. We have included contributions containing the irrelevant fields $\Phi_{P}^{\delta}(x)$ since fields with $P=\mathcal{O}(\delta)$ are nearly marginal. Independence of the correlation functions from the cut-off $\epsilon$ follows if the coupling constants satisfy the RG flow equations:

$$
\begin{aligned}
& \epsilon \frac{d}{d \epsilon} u-\delta^{2} u=-f_{\vartheta \vartheta}^{\vartheta} u^{2}-2 \int_{0}^{\infty} d P_{1} f_{\vartheta P_{1}}^{\vartheta} \lambda\left(P_{\mathbf{1}}\right) u-\int_{0}^{\infty} d P_{2} d P_{1} f_{P_{2} P_{1}}^{\vartheta} \lambda\left(P_{1}\right) \lambda\left(P_{2}\right) \\
& \epsilon \frac{d}{d \epsilon} \lambda(P)+P^{2} \lambda(P)=-F_{\vartheta \vartheta}^{P} u^{2}-2 \int_{0}^{\infty} d P_{1} F_{\vartheta P_{1}}^{P} \lambda\left(P_{1}\right) u-\int_{0}^{\infty} d P_{2} d P_{1} F_{P_{2} P_{1}}^{P} \lambda\left(P_{1}\right) \lambda\left(P_{2}\right)
\end{aligned}
$$

Let us analyze the equations at or near the new fixed point. Equations (4.24), (4.16), (4.17) then suggest that $\lambda(P)$ must be peaked for small $P$. Plugging in the approximate formulae (4.16), (4.17) one simplifies the RG flow equations to

$$
\begin{aligned}
\epsilon \frac{d}{d \epsilon} u & =\delta^{2} u-2 \delta w^{2}, \\
\epsilon \frac{d}{d \epsilon} \lambda(P) & =-P^{2} \lambda(P)-\frac{1}{2 \pi} \frac{4 P^{2}}{\delta^{2}+P^{2}} w^{2},
\end{aligned}
$$

where

$$
w \equiv u+\int_{0}^{\infty} d P \lambda(P)
$$

We may observe that the RG flow equations have a fixed point $\lambda_{*}(P)$. First note that one can determine the $P$-dependence of $\lambda_{*}(P)$ for $P \in \mathbb{R}_{+}$from (4.26) and (4.27) to be

$$
\lambda_{*}(P)=\frac{2}{\pi} \frac{\delta v_{*}}{\delta^{2}+P^{2}}, \quad v_{*} \equiv \int_{0}^{\infty} d P \lambda_{*}(P) .
$$

Inserting this into (4.26) yields the following equations for the fixed point values $u_{*}$ and $v_{*}$ :

$$
\begin{aligned}
& 0=\delta u_{*}-2\left(u_{*}+v_{*}\right)^{2}, \\
& 0=-\delta v_{*}-\left(u_{*}+v_{*}\right)^{2} .
\end{aligned}
$$

This implies in particular that

$$
u_{*}=2 \delta, \quad v_{*}=-\delta, \quad w_{*}=u_{*}+v_{*}=\delta,
$$

are the coupling constants at the new fixed point of the renormalization group. 
Determination of the new fixed point

We will next determine the perturbed boundary state $\left|B_{*}\right\rangle$ at the new RG fixed point. We claim that

$$
\left|B_{*}\right\rangle_{\mathrm{L}}^{\text {pert }}=\left|B_{-\delta}\right\rangle_{\mathrm{L}} .
$$

In order to verify (4.32) let us first note that the new fixed point must have the same value of $\mu_{\mathrm{B}}$. This can be seen as follows. We had previously seen that the wave-function of the boundary state $B_{\delta}\left(\phi_{0}\right)$ approaches a constant for $\phi_{0} \rightarrow-\infty$. Subleading contributions for $\phi_{0} \rightarrow-\infty$ that decay exponentially will produce poles in amplitudes such as $A_{P}^{\delta}=\left\langle P \mid B_{\delta}\right\rangle_{\mathrm{L}}$ which come from the asymptotic behavior of the integrand in (4.8). The next-to leading order contribution to $B_{\delta}\left(\phi_{0}\right)$ is of the order $e^{\phi_{0}}$ which corresponds to the pole of $A_{P}^{\delta}$ at $P=-i / 2$, see (4.5). It is important to note that this contribution is proportional to $\mu_{\mathrm{B}}$, as may also be inferred from (4.5). Note, however, that the perturbing boundary field $\Phi_{\vartheta}^{\delta}(x)$ behaves asymptotically as $e^{(1+\delta) \phi_{0}}$. Indeed, a generic boundary field $\Phi_{i \varpi}^{\delta}(x)$ has asymptotic behavior of the form given in equation (4.13). The first term in (4.13) vanishes in the case of the perturbing boundary field $\Phi_{\vartheta}^{\delta}(x)$ as follows from $C_{\delta}(i \delta)=0$. This means that the perturbative expansion of $\left|B_{\delta}\right\rangle_{\mathrm{L}}^{\text {pert }}$ in powers of $\lambda$ will only generate terms which vanish faster than $e^{\phi_{0}}$ when $\phi_{0} \rightarrow \infty$. The value of $\mu_{\mathrm{B}}$ must therefore be unchanged.

In order to further confirm our prediction (4.32) let us calculate, to lowest order in $\delta$, the deviation $d_{*}(P)$ :

$$
d_{*}(P)=\left\langle P \mid B_{*}\right\rangle_{\mathrm{L}}-\left\langle P \mid B_{\delta}\right\rangle_{\mathrm{L}}
$$

We find

$$
\begin{aligned}
d_{*}(P) \underset{\delta \rightarrow 0}{\sim} & -2 \pi u_{*}\left\langle P\left|\Phi_{\vartheta}^{\delta}(0)\right| B_{\delta}\right\rangle_{\mathrm{L}} \\
& -2 \pi \int_{0}^{\infty} d P^{\prime} \lambda_{*}\left(P^{\prime}\right)\left\langle P\left|\Phi_{P^{\prime}}^{\delta}(0)\right| B_{\delta}\right\rangle_{\mathrm{L}} .
\end{aligned}
$$

The relevant bulk-boundary correlation functions are given in equation (4.18). Note that they are independent of $P^{\prime}$. We may therefore carry out the integral over $P^{\prime}$ in (4.34) by using the definition of $v_{*}$ given in (4.28). It follows that

$$
d_{*}(P) \underset{\delta \rightarrow 0}{\sim}-2 \pi w_{*}\left\langle P\left|\Phi_{\vartheta}^{\delta}(0)\right| B_{\delta}\right\rangle_{\mathrm{L}} .
$$

By using equations (5.33) and (4.18) we arrive at the following formula for the perturbed one point function at the new fixed point:

$$
d_{*}(P) \underset{\delta \rightarrow 0}{\sim}-\delta \frac{2 \pi P}{\sinh 2 \pi P} \Theta(P) .
$$

This is the same result as one would have found from (4.32) by keeping terms up to $\mathcal{O}(\delta)$.

The time-independent treatment described in this section may lead one to conjecture that there should exist a time-dependent solution of string theory which interpolates between asymptotic states that are D1 branes with labels $\delta$ and $-\delta$ respectively. This is what we are going to construct in the following section. 


\section{A noncritical time-dependent string background}

We now want to apply the formalism developed in section 3 to the case that the $\mathrm{CFT}_{\mathrm{S}}$, the conformal field theory which describes the spatial part of the background is (boundary) Liouville theory with $c=25$. The boundary action which will define the perturbed time-dependent background is then given by the expression

$$
S_{\mathrm{Bd}} \equiv \lambda \int_{\partial \Sigma} d x\left[e^{\delta X_{0}} \Phi_{\vartheta}^{\delta}\right](x)
$$

Note that the short distance singularities in the OPE of $\left[e^{\delta X_{0}} \Phi_{\vartheta}^{\delta}\right](x)$ with itself are integrable. It follows that (3.32) indeed defines a conformal boundary state to all orders in a formal expansion in the parameter $\lambda e^{\delta t}$.

In order to describe the time evolution of the perturbed boundary state $\left.\left|B_{\delta}\right\rangle\right\rangle_{\text {dyn }}$ we will consider the amplitude

$$
A(P, t) \equiv\left\langle\left\langle P, t \mid B_{\delta}\right\rangle_{\mathrm{dyn}}, \quad\left\langle\langle P , t | \equiv \langle P | \otimes \left\langle\left. t\right|_{\mathrm{x}_{0}},\right.\right.\right.
$$

from which the corresponding amplitude

$$
A(P, \omega) \equiv\left\langle\left\langle P, \omega \mid B_{\delta}\right\rangle_{\mathrm{dyn}}, \quad\left\langle\langle P , \omega | \equiv \langle P | \otimes \left\langle\left.\omega\right|_{\mathrm{x}_{0}}\right.\right.\right.
$$

will then follow by Fourier transformation.

\subsection{RG improvement in the case of a near-marginal continuum}

In order to employ the technique from section 2 to our specific example let us assume having introduced a proper regularization scheme with a short-distance cut-off $\epsilon$. We will consequently have to work with a renormalized boundary action density $S_{\mathrm{Bd}}^{\mathrm{ren}}(x)$ which will be of the form

$$
S_{\mathrm{Bd}}^{\mathrm{ren}}(x)=\sum_{n=1}^{\infty}\left(U_{n} \epsilon^{\left(n^{2}-1\right) \delta^{2}}\left[e^{n \delta X_{0}} \Phi_{\vartheta}^{\delta}\right](x)+\int_{0}^{\infty} d P \lambda_{n}(P) \epsilon^{n^{2} \delta^{2}+P^{2}}\left[e^{n \delta X_{0}} \Phi_{P}^{\delta}\right](x)\right) .
$$

The conditions for the $\epsilon$-independence of the correlation functions are then found to be the equations

$$
\begin{aligned}
& \epsilon \frac{d}{d \epsilon} U_{n}+\left(n^{2}-1\right) \delta^{2} U_{n}= \\
& =\sum_{m=1}^{n-1}\left(-f_{\vartheta \vartheta}^{\vartheta} U_{m} U_{n-m}-2 \int_{0}^{\infty} d P_{\mathbf{1}} f_{\vartheta P_{\mathbf{1}}}^{\vartheta} U_{m} \lambda_{n-m}\left(P_{\mathbf{1}}\right)-\int_{0}^{\infty} d P_{2} d P_{\mathbf{1}} f_{P_{2} P_{1}}^{\vartheta} \lambda_{m}\left(P_{\mathbf{1}}\right) \lambda_{n-m}\left(P_{2}\right)\right) \\
& \epsilon \frac{d}{d \epsilon} \lambda_{n}(P)+\left(n^{2} \delta^{2}+P^{2}\right) \lambda_{n}(P)= \\
& =\sum_{m=1}^{n-1}\left(-F_{\vartheta \vartheta}^{P} U_{m} U_{n-m}-2 \int_{0}^{\infty} d P_{\mathbf{1}} F_{\vartheta P_{\mathbf{1}}}^{P} U_{m} \lambda_{n-m}\left(P_{\mathbf{1}}\right)-\int_{0}^{\infty} d P_{2} d P_{\mathbf{1}} F_{P_{2} P_{1}}^{P} \lambda_{m}\left(P_{\mathbf{1}}\right) \lambda_{n-m}\left(P_{2}\right)\right) .
\end{aligned}
$$


Removing the cut-off $\epsilon$ by sending $\epsilon \rightarrow 0$ should reproduce the bare action (3.33). This means that we are interested in the solution to (5.5) which is defined by the following supplementary conditions:

$$
\begin{aligned}
& \lim _{\epsilon \rightarrow 0} \lambda_{n}(P) \epsilon^{n^{2} \delta^{2}+P^{2}}=0 \text { for } P \in \mathbb{R}_{+}, \\
& \lim _{\epsilon \rightarrow 0} U_{n} \epsilon^{\left(n^{2}-1\right) \delta^{2}}=0 \text { for } n>1, \\
& \lim _{\epsilon \rightarrow 0} U_{1}=\nu \delta .
\end{aligned}
$$

$\lambda \equiv \nu \delta$ is the value of the corresponding "bare" coupling introduced in (3.32). It will again turn out to be useful to measure $\lambda$ in units of $\delta$ by introducing $\nu=\lambda / \delta$.

Equations (5.5), (5.7) can be solved recursively. It is easy to see that we must have $U_{1}=\lambda$ and $\lambda_{1}(P)=0$ for $P \in \mathbb{R}_{+}$. For $n>1$ let us note that a special solution to the inhomogenous equations (5.5) is always given by $\epsilon$-independent coupling constants $\lambda_{n}(P)$. The general solution of the equations (5.5) is then obtained by adding an arbitrary solution to the homogeneous equations which are obtained from (5.5) by dropping the right hand side. However, the solutions to these homogeneous equations will never satisfy the boundary conditions (5.7) unless they are identically zero. We therefore find that $\lambda_{n}(P)$ is determined for $n \geq 2$ from the recursion relations

$$
\begin{aligned}
& \left(n^{2} \delta^{2}+P^{2}\right) \lambda_{n}(P)= \\
& -\sum_{m=1}^{n-1}\left(F_{\vartheta \vartheta}^{P} U_{m} U_{n-m}+2 \int_{0}^{\infty} d P_{\mathbf{1}} F_{\vartheta P_{1}}^{P} U_{m} \lambda_{n-m}\left(P_{\mathbf{1}}\right)+\int_{0}^{\infty} d P_{2} d P_{\mathbf{1}} F_{P_{2} P_{1}}^{P} \lambda_{m}\left(P_{\mathbf{1}}\right) \lambda_{n-m}\left(P_{2}\right)\right) .
\end{aligned}
$$

It follows that $\lambda_{n}(P) \propto\left(n^{2} \delta^{2}+P^{2}\right)^{-1}$ is strongly peaked around $P=\mathcal{O}(\delta)$. Noting that the OPE coefficients $F_{P_{2} P_{1}}^{P}$ are approximately constant in this region, cf. eqn. (4.16), allows us to get the $\delta \rightarrow 0$ asymptotics of the integrations over $P_{2}, P_{1}$ by introducing a rescaled momentum variable

$$
q=P / \delta
$$

and substituting the asymptotic values (4.16) at fixed $q_{i}$ 's into (5.8). Define

$$
V_{n} \equiv \int_{0}^{\infty} d P \lambda_{n}(P)
$$

One finds then from (5.8), (4.16) that the $P$-dependence of $\lambda_{n}(P)$ (at $\delta \rightarrow 0$ ) can be written in the form

$$
\lambda_{n}(P)=\lambda_{n}(q \delta)=\frac{V_{n}}{\delta} \frac{n+1}{n^{2}+q^{2}} \frac{4 q^{2}}{2 \pi\left(1+q^{2}\right)} .
$$

One further finds that (5.5) and (5.6) lead to a closed set of equations for the coupling constants $V_{n}$ and $U_{n}$,

$$
\begin{aligned}
\left(n^{2}-1\right) \delta U_{n} & =-2 R_{n}, \\
(n+1) \delta V_{n} & =-R_{n},
\end{aligned} \quad R_{n} \equiv \sum_{m=1}^{n-1}\left(U_{m} U_{n-m}+2 U_{m} V_{n-m}+V_{m} V_{n-m}\right) .
$$


These recursion relations combined with the initial conditions

$$
U_{1}=\delta \nu, \quad V_{1}=0
$$

completely determine the coupling constants $U_{n}$ and $\lambda_{n}(P)$ via (5.11). It is easy to show that (5.12) and (5.13) imply that $U_{n}=\mathcal{O}(\delta)$ and $V_{n}=\mathcal{O}(P)$. The integral over $P$ in (5.4) will also be of the order $\delta$ since $\lambda_{n}(P)$ is peaked around $P=\mathcal{O}(\delta)$. In order to extract the leading behavior of $\left.\left|B_{\delta}\right\rangle\right\rangle_{\text {dyn }}$ for $\delta \rightarrow 0$ we may therefore indeed work with the renormalized action (5.4) in the following.

\subsection{Perturbed one point function}

As a simple example for the application of our findings let us now consider the amplitude $A(P, t)$. It will be convenient to subtract the constant initial value of this quantity and consider

$$
D(P, t) \equiv\left\langle\left\langle P, t \mid B_{\delta}\right\rangle_{\mathrm{dyn}}-\left\langle\left\langle P, t \mid B_{\delta}\right\rangle\right\rangle_{\mathrm{stat}} .\right.
$$

According to the discussion in our previous subsection we may calculate this quantity to leading order in $\delta$ by expanding (5.4) to the first order,

$$
\begin{aligned}
D(P, t) \underset{\delta \rightarrow 0}{\sim} & -2 \pi U(t)\left\langle P\left|\Phi_{\vartheta}^{\delta}(0)\right| B_{\delta}\right\rangle_{\mathrm{L}} \\
& -2 \pi \int_{0}^{\infty} d P^{\prime} \lambda_{P^{\prime}}(t)\left\langle P\left|\Phi_{P^{\prime}}^{\delta}(0)\right| B_{\delta}\right\rangle_{\mathrm{L}},
\end{aligned}
$$

where we have used the notations

$$
\lambda_{P}(t)=\sum_{n=1}^{\infty} e^{\delta n t} \lambda_{n}(P), \quad U(t)=\sum_{n=1}^{\infty} e^{\delta n t} U_{n}
$$

The relevant correlators are given in equation (4.18). Note that they are independent of $P^{\prime}$. We may therefore carry out the integral over $P^{\prime}$ in (5.15) as follows:

$$
\int_{0}^{\infty} d P^{\prime} \lambda_{P^{\prime}}(t)=\sum_{n=1}^{\infty} e^{\delta n t} \int_{0}^{\infty} d P^{\prime} \lambda_{n}\left(P^{\prime}\right)=\sum_{n=1}^{\infty} e^{\delta n t} V_{n} \equiv V(t) .
$$

Inserting the explicit expression (4.18) leads to

$$
D(P, t) \underset{\delta \rightarrow 0}{\sim}-W(t) \frac{2 \pi P}{\sinh 2 \pi P} \Theta(P), \quad W(t) \equiv U(t)+V(t) .
$$

The task remains to calculate $W(t)$ explicitly. Note that so far we had to assume that $t$ is sufficiently small. We will indeed see that the range of convergence of the series (5.16) is finite. However, the function $W(t)$ will turn out to have an analytic continuation which allows us to extend the definition of $D(P, t)$ to all real values of $t$. 


\subsection{Time evolution}

The recursion relations (5.12) are easily translated into differential equations for the generating functions (5.16),

$$
\begin{aligned}
& \delta^{-1} \ddot{U}=\delta U-2 W^{2}, \\
& \dot{V}=-\delta V-W^{2},
\end{aligned}
$$

The dots indicate derivative with respect to $t$. In this section we will find the explicit solutions to the time-evolution equations (5.19) with the boundary conditions (5.13). This will then allow us, using (5.11), to find the function $\lambda_{P}(t)$.

To begin with, let us note that the time evolution equations (5.19) can be rewritten in the form

$$
\ddot{U}=-2 \delta \frac{\partial \mathcal{P}}{\partial U}, \quad \dot{V}=-\frac{\partial \mathcal{P}}{\partial V}
$$

where

$$
\mathcal{P}=\mathcal{P}(U, V)=-\frac{\delta}{4} U^{2}+\frac{\delta}{2} V^{2}+\frac{1}{3}(U+V)^{3} .
$$

The existence of such a "potential" function $\mathcal{P}(U, V)$ can be traced back to the cyclic symmetry of the OPE coefficients. However equations (5.20) are clearly not the standard Euler-Lagrange equations for two mechanical degrees of freedom due to the first order derivative term $\dot{V}$. The dissipative nature of this system of equations can be discerned by looking at the time derivative

$$
\frac{d}{d t}\left[\frac{1}{2} \dot{U}^{2}+\mathcal{P}(U, V)\right]=-(\dot{V})^{2} \leq 0 .
$$

Here in the square brackets we have an expression that can be thought of as an effective energy for the $U$-degree of freedom which ought to monotonically decrease due to this identity.

Although as we have just demonstrated the energy is no longer an integral of motion for equations (5.20), there is another integral of motion. One can easily show that

$$
\frac{d}{d t}\left[e^{\delta t}\left(\delta^{-1} \dot{U}-U-2 V\right)\right]=0
$$

Using the initial conditions at $t \rightarrow-\infty$, that are essentially given by the fact that our solutions in that limit can be represented as power series (5.16), we see that the integral of motion in (5.23) assumes zero value. This reduces (5.20) to a system of first order differential equations

$$
\begin{aligned}
& \dot{U}=\delta(U+2 V) \\
& \dot{V}=-\delta V-(U+V)^{2} .
\end{aligned}
$$

One further observes that the $W=U+V$ degree of freedom decouples:

$$
\dot{W}=\delta W-W^{2} .
$$

Solving this equation we obtain

$$
W(t)=\frac{\delta e^{\delta t}}{C+e^{\delta t}},
$$


where $C$ is a constant of integration. Substituting (5.26) back into (5.24) we obtain

$$
\begin{aligned}
& U(t)=2 \delta\left[1-C e^{-\delta t} \ln \left(1+C^{-1} e^{\delta t}\right)\right]=\delta \sum_{n=1}^{\infty}(-1)^{n+1} e^{n \delta t} C^{-n}, \\
& V(t)=W(t)-U(t)=\delta \sum_{n=2}^{\infty}(-1)^{n}\left(\frac{1-n}{1+n}\right) C^{-n} e^{n \delta t}
\end{aligned}
$$

The integration constant $C$ is found to be related to the bare coupling $\lambda=\nu \delta$ by observing that (5.27) implies $U_{1}=\delta C^{-1}$. Taking into account the condition (5.7) therefore yields the relation

$$
C=\delta \lambda^{-1}=\nu^{-1}
$$

We are left with the task to calculate $\lambda_{P}(t)$ explicitly. Plugging the coefficients $V_{n}$ from (5.28) into (5.11) we obtain

$$
\begin{aligned}
& \lambda(q, t) \equiv \lambda_{q \delta}(t)=\sum_{n=1}^{\infty} \lambda_{n}(q \delta) e^{n \delta t}=\frac{2 q^{2}}{\pi\left(1+q^{2}\right)} \tilde{\lambda}(q, t), \\
& \tilde{\lambda}(q, t)=\sum_{n=2}^{\infty} \frac{1-n}{n^{2}+q^{2}}\left(-\nu e^{\delta t}\right)^{n} .
\end{aligned}
$$

The function $\tilde{\lambda}_{P}(t)$ can be represented as

$$
\tilde{\lambda}(q, t)=f_{q}(t)-\delta^{-1} \frac{d}{d t} f_{q}(t)
$$

where

$$
\begin{array}{r}
f_{q}(t)=-\frac{\nu e^{\delta t}}{2 q\left(q^{2}+1\right)}\left[(q-i){ }_{2} F_{1}\left(1-i q, 1 ; 2-i q ;-\nu e^{\delta t}\right)+\right. \\
\left.+(q+i){ }_{2} F_{1}\left(1+i q, 1 ; 2+i q ;-\nu e^{\delta t}\right)\right] .
\end{array}
$$

Formulas (5.30), (5.31), (5.32) provide explicit expressions for the time-dependent couplings $\lambda_{P}(t)$ via hypergeometric functions.

\subsection{Asymptotics $t \rightarrow \infty$}

Having found the explicit expression (5.26) for the function $W(t)$ we may now return to the discussion of the perturbed one point function $A(P, t)$. Observing that the expression (5.26) is well-defined for all values of $t$ will not only allow us to to discuss the asymptotics of $A(P, t)$ for $t \rightarrow \infty$, it will also finally give us the leading order result for the amplitude $A(P, \omega)$.

To begin with, let us observe that the asymptotic values

$$
\lim _{t \rightarrow \infty} U(t)=2 \delta=u_{*}, \quad \lim _{t \rightarrow \infty} V(t)=-\delta=v_{*}, \quad \lim _{t \rightarrow \infty} W(t)=\delta=w_{*},
$$

coincide with the fixed point values $u_{*}, v_{*}$ and $w_{*}$ that we had found in the time-independent treatment of subsection (4.4). The time evolution of $U$ and $V$ therefore smoothly interpolates 
between the values of the corresponding couplings at the UV and IR fixed points in the timeindependent picture. The corresponding asymptotic values of $A(P, t)$ can be identified with the overlaps $\left\langle P \mid B_{\delta}\right\rangle_{\mathrm{L}}$ and $\left\langle P \mid B_{*}\right\rangle_{\mathrm{L}}$, respectively. Let us finally remark that the values $u_{*}, v_{*}$ correspond to a local minimum of the "potential" function $\mathcal{P}(\underline{5.21})$.

It will also be quite suggestive to look at the asymptotics of $\lambda(q, t)$ for $t \rightarrow \infty$. It can be deduced from the asymptotic of the hypergeometric function for $|x| \rightarrow \infty$,

$$
{ }_{2} F_{1}(1-i q, 1 ; 2-i q ; x) \underset{|x| \rightarrow \infty}{\rightarrow}(-x)^{-1} \frac{q+i}{q}+(-x)^{-1-i q} \Gamma(2-i q) \Gamma(i q) .
$$

Using this asymptotics we obtain

$$
\lambda(q, t) \rightarrow-\frac{2}{\pi\left(1+q^{2}\right)}+\frac{1}{\sinh (\pi q)}\left(e^{i q \delta t} \nu^{i q} \frac{q}{1+i q}+e^{-i q \delta t} \nu^{-i q} \frac{q}{1-i q}\right)
$$

The first term, which is time independent, coincides with the fixed point function $\lambda_{*}(P)$ given in (4.28), (4.31). Together with the asymptotic values (5.33) this implies that the stationary part of the $t \rightarrow \infty$ asymptotics of the coupling constants corresponds to the fixed point found in subsection 4.4 which we had identified with the D1 branes labelled by the parameter $-\delta$. The oscillatory part of (5.35) corresponds to a perturbation of this fixed point background by marginal operators $\Phi_{P}^{\delta} e^{ \pm i P X_{0}}$ with appropriate coupling constants. This time-dependent piece can be interpreted as open string radiation on top of the D1 brane with label $-\delta$.

In order to round off the discussion let us finally calculate $D(P, \omega) \equiv\left\langle\left\langle P, \omega \mid B_{\delta}\right\rangle_{\text {dyn }}-\right.$ $\left\langle\left\langle P, \omega \mid B_{\delta}\right\rangle\right\rangle_{\text {stat }}$. We simply have to perform the inverse Fourier transformation from $\left\langle\left. t\right|_{\mathrm{X}_{0}}\right.$ to $\left\langle\left.\omega\right|_{\mathrm{X}_{0}}\right.$ by using

$$
\widehat{W}(\omega) \equiv \int_{\mathbb{R}} \frac{d t}{2 \pi} e^{i \omega t} W(t)=\frac{i \nu^{-i \frac{\omega}{\delta}}}{2 \sinh \left(\pi \frac{\omega}{\delta}\right)} .
$$

We arrive at the expression

$$
D(P, \omega) \underset{\delta \rightarrow 0}{\sim}-\frac{i \nu^{-i \frac{\omega}{\delta}}}{2 \sinh \left(\pi \frac{\omega}{\delta}\right)} \frac{2 \pi P}{\sinh 2 \pi P} \Theta(P),
$$

from which the closed string emission in the decay of a D1 brane with parameter $\delta$ into a D1 brane with parameter $-\delta$ can be calculated.

\section{Discussion}

In this paper we analyzed a particular model of D1-brane decay in non-critical $c=1$ string theory. The presence of a small parameter $\delta$ responsible for the mass of the tachyon allowed us to analyze quantitatively some features of the time-dependent CFT that describes the tachyon condensation. In particular, employing the RG-resummation technique, we found the boundary state for the model in the leading order in the $\delta$-expansion. We could show that in the $t \rightarrow \infty$ limit the time-dependent CFT looks like a certain static background describing another D1brane perturbed by a time-dependent marginal perturbation describing an open string radiation 
propagating to infinity. We showed that the static D1-brane background at hand coincides with the end point of the RG evolution triggered by perturbing the Liouville part of the theory by the relevant operator corresponding to the tachyon.

The issue of what is the relation between the RG flow triggered by a relevant operator corresponding to the tachyon and the description of its condensation by a time-dependent CFT was recently addressed in [FHL] in the framework of closed string theory. The RG-equations are first order in the RG "time" and have a dissipative nature as demonstrated in general by Zamolodchikov's $c$-theorem. On the other hand the time-evolution equations in target space are (at least quasiclassically) second order in time and, superficially, preserve the total space-time energy. This seems naively to preclude any simple qualitative relationship between the two. It was shown however in [FHL] that if one properly accounts for dilaton couplings a damping force appears for the time-evolution equations for the rest of the couplings. The last feature makes it in principle possible for the time evolution to end in an RG fixed point accompanied by a time-dependent dilaton and some examples of this situation were discussed in [FHL].

In the case of open string condensation no time-dependent dilaton couplings enter the consideration at least at tree level. For the model studied in the present paper a different type of relationship with RG flows was found. In this case the time-dependent CFT describes a localized tachyonic degree of freedom interacting with a continuum of open string scattering states. With the total energy being preserved the tachyonic degree of freedom relaxes asymptotically into the RG fixed point at the expense of producing open string radiation that escapes to infinity.

It seems natural to expect that generalizations of this mechanism will be applicable in a wide range of situations where tachyons are localized in a non-compact target space and the boundary conditions at infinity do not change in the course of tachyon condensation. In the present case the dominant decay channel was open string radiation, but in other cases like the example discussed in [T2] one will find that most of the energy is carried away by closed string radiation.

For the cases in which the tachyon condensation produces changes of the boundary conditions at infinity the world sheet description is expected to be more subtle (see [SeibSh] for a general discussion and [T2] for some related observations concerning time-dependent phenomena). Reaching the new fixed point requires giving an expectation value to a non-normalizable operator that seems to be hard to generate (at least perturbatively) from localized tachyons that are normalizable modes. One should also note that the very notion of radiation becomes problematic in such cases. In the case of the model of unstable D1 branes studied in this paper the new fixed point has the same value of $\mu_{B}$ and thus does not involve changing the boundary conditions at infinity [T2].

It may happen that an unstable system of D-branes decays into a state not carrying any open string scattering states. In that case closed string radiation may be the dominant mode of decay. For the present model the closed string radiation appears as a subdominant effect proportional to the string coupling constant. It would be interesting however to study back reaction effects on the open string tachyon condensation due to the closed string radiation for the present model. We leave this issue for future work. 
Another question that is worth clarification is a precise relation between the oscillatory piece of the asymptotic (5.35) and open string pair creation as may be measured by a suitable twopoint function. This will require constructing marginal operators in the time-dependent theory and finding their decomposition into in-coming and out-going scattering states. We are planning to address these questions in future work.

At the technical level the present model may look very similar to localized closed string tachyons in $\mathbb{C} / \mathbb{Z}_{N}$ non-supersymmetric orbifolds of critical superstrings (see [APS] for the initial discussion) for large values of $N$. The tachyon in those models lives in the twisted sector and describes a localized degree of freedom. Its mass goes to zero when $N$ becomes very large so it looks like one has at his/her disposal a small parameter similar to $\delta$ in the present model. It may therefore look appealing to apply methods of the present paper to those models in the $N \rightarrow \infty$ limit. There is however an important distinction between the $\mathbb{C} / \mathbb{Z}_{N}$ theories and models analogous to the one studied in this paper. The tachyon condensation in those models does involve changing boundary conditions at infinity. Furthermore the world sheet analysis of [SarSat $]$, [DIR] seems to indicate that the final fixed point for the RG flow is not reachable through the $1 / N$ expansion. This unpalatable feature may also be linked to the change of boundary conditions at infinity. It seems to be important to understand better how to handle these models from the world sheet point of view.

\section{Acknowledgments}

A. K. and J. T. would like to thank Kavli Institute for Theoretical Physics, where their collaboration on this project started, for warm hospitality. Part of this work was done during visits of K. G. and A. K. to DESY Theory Group whose hospitality is gratefully acknowledged. A.K. wants to thank Davit Sahakyan for useful discussions. The work of A.K. was supported in part by DOE grant DE-FG02-96ER40949. The work of K.G. was supported by the EUCLID Network, contact number HPRN-CT-2002-00325. 


\section{A. Perturbations with UV divergences}

To simplify the discussion in sections 2 and 3 we had been assuming the absence of UV divergencies. However, it will not be hard to show that the main results carry over to the case when there are UV divergencies which arise from the presence of the identity field in some operator product expansions.

\section{A.1 Comments on the time-independent case}

As a typical example of where UV divergences would appear, consider a CFT with discrete spectrum and let our near marginal field fuse with itself to give the identity,

$$
\phi_{0}(\tau) \phi_{0}(0)=C_{00} \mathbb{1}\left(2 \sin \frac{\tau}{2}\right)^{-2+2 y_{0}} \mathbb{1}+\text { other terms } .
$$

More generally, we can consider the field fusing to create many non-nearly marginal relevant fields. To regulate these divergences one simply repeats the steps of section 2.2. A subtlety comes in specifying renormalisation conditions to replace (2.15) which will no longer make sense in general. One choice is to introduce a renormalisation scale $\Lambda$ and define physical couplings $\hat{\mu}_{k}$ via $\hat{\mu}_{k}=\mu_{k}(\Lambda)$. Singular terms in the expression $\mu_{k}(\epsilon)$ will then cancel similar singularities in the perturbation expansion such that the resulting correlation functions are finite and independent of $\epsilon$.

However, through all this the renormalisation group equations (2.14) are unchanged. It then follows that if all couplings are assumed to be small and the system flows to a non-trivial fixed point of the RG-equations, the couplings to non-nearly marginal fields will be $\mathcal{O}\left(\delta^{2}\right)$ and so can be ignored to this level in the analysis. Having reduced the system to that involving only nearly-marginal fields, the leading $\delta$-behaviour of the remaining couplings $\mu_{k}(\epsilon)$ is independent of $\epsilon$ and the results of the text apply.

In conclusion, even in the presence of UV divergences involving non-nearly marginal fields, the leading behaviour of correlation functions is given by (2.23) wherein $\mu_{k}(\epsilon)$ can be calculated from the renormalisation group equations involving only nearly marginal fields. In the case where the UV divergences come from nearly marginal fields, things are more complicated and we have nothing to say at this time.

\section{A.2 Time-dependent perturbations in the presence of divergencies}

We would like to convince ourselves that the main features of the discussion in subsection 3.2 are still valid if there are divergencies in the perturbative expansion.

We will assume that the fields $\phi_{a}$ in the complement $N=F \backslash M$ of the set of all marginal fields $M$ are all such that $y_{a}=\mathcal{O}(1)$ when $\delta \rightarrow 0$. We use the letters $a, b, c, \ldots$ to label the elements of $N . \delta^{2}$ will be identified with the largest possible value that $y_{i}$ takes for $\phi_{i} \in M$. To simplify life we will furthermore assume that all relevant OPE coefficients $C_{i j}^{k}, C_{a j}^{k}, C_{a b}^{k}, C_{i j}^{c}$, $C_{a j}^{c}$ and $C_{a b}^{c}$ are of order $\mathcal{O}(1)$ when $\delta \rightarrow 0$. 
We are then led to consider the regulated perturbation,

$$
\begin{aligned}
S_{\text {reg }}=\sum_{n=1}^{\infty} & {\left[\sum_{k \in M} u_{k, n} \epsilon^{n^{2} \delta^{2}-y_{k}} \int d x\left[e^{n \delta X^{0}} \phi_{k}\right](x)\right.} \\
& \left.+\sum_{a \in N} v_{a, n} \epsilon^{n^{2} \delta^{2}-y_{a}} \int d x\left[e^{n \delta X^{0}} \phi_{a}\right](x)\right] .
\end{aligned}
$$

Following the arguments in subsection 3.2 now leads to the following recursion relations

$$
\begin{aligned}
& \left(y_{k}-n^{2} \delta^{2}\right) u_{k, n}= \\
& \quad=\sum_{m=1}^{n-1}\left[\sum_{a, b \in N} C_{a b}{ }^{k} v_{a, m} v_{b, m}+2 \sum_{a \in N} \sum_{i \in M} C_{a i}{ }^{k} v_{a, m} u_{i, n-m}+\sum_{i, j \in M} C_{i j}{ }^{k} u_{i, m} u_{j, n-m}\right], \\
& \left(y_{c}-n^{2} \delta^{2}\right) v_{c, n}= \\
& =\sum_{m=1}^{n-1}\left[\sum_{a, b \in N} C_{a b}{ }^{c} v_{a, m} v_{b, m}+2 \sum_{a \in N} \sum_{i \in M} C_{a i}{ }^{c} v_{a, m} u_{i, n-m}+\sum_{i, j \in M} C_{i j}{ }^{c} u_{i, m} u_{j, n-m}\right] .
\end{aligned}
$$

These equations are supplemented with the boundary conditions

$$
u_{k, 1}=\kappa_{k} \delta^{2}, \quad u_{k, 1}=0, \quad v_{a, 1}=0
$$

Keeping in mind that $y_{c}=\mathcal{O}(1)$ we may easily deduce from (A.3), A.4 that $u_{k, n}=\mathcal{O}\left(\delta^{2}\right)$, but $v_{n}=\mathcal{O}\left(\delta^{4}\right)$. Working to leading order in $\delta^{2}$ we may therefore simplify (A.3) to

$$
u_{k, n}=\frac{1}{y_{k}-n^{2} \delta^{2}} \sum_{m=1}^{n-1} \sum_{i, j \in M} C_{i j}{ }^{k} u_{i, m} u_{j, n-m} .
$$

It follows that the generating functions $U_{k}(t)=\sum_{n=1}^{\infty} u_{k, n} e^{n \delta t}$ satisfy the same time evolution equations (3.47) as before, and that the couplings $v_{a, n}$ do not modify the leading order result (3.50) for the correlation functions. However, the couplings $\hat{v}_{a, n}=v_{a, n} \epsilon^{n^{2} \delta^{2}-y_{a}}$ may diverge when $\epsilon \rightarrow 0$. These divergencies cancel the divergencies which would arise in the perturbative integrals when removing the cut-off $\epsilon$.

As pointed out in subsection 3.2, it is not clear in general if the motion described by the time evolution equations (3.47) will remain bounded. It will certainly not remain bounded if the set $N$ contains relevant fields not equal to the identity and if some of the corresponding couplings $v_{a, 1}$ do not vanish. The perturbative approach to the construction of amplitudes in the time-dependent theory will then break down after a certain time $t$. However, at least in the cases where the only relevant field contained in $N$ is the identity and where the couplings $U_{k}(t)$ determined from the time evolution equations (3.47) stay bounded, one may reliably use our formalism to calculate the one point functions in the time dependent background. 


\section{B. Aspects of boundary Liouville theory}

Certain results on boundary Liouville theory play an important role in our paper. The relevant results include the approximate expression (4.16) for the operator product coefficients $F_{P_{2} P_{1}}^{P_{3}}$ of the boundary fields $\Phi_{P}^{\delta}(x)$ respectively, as well as the relation (4.12) between bulk and boundary fields. This appendix is devoted to the derivation of these results.

The boundary fields $\Phi_{P}^{\delta}(x)$ used in this paper are related to the more general boundary fields $\Psi_{\beta}^{\sigma_{2} \sigma_{1}}(x)$ studied in $[\overline{\mathrm{PT}}]$ by a change of normalization,

$$
\Phi_{P}^{\delta}(x)=g_{\beta}^{\sigma \sigma} \Psi_{\beta}^{\sigma \sigma}(x), \quad\left\{\begin{array}{l}
\beta=1+i P \\
2 \sigma=1-\delta
\end{array}\right\}
$$

The expression for $g_{\beta}^{\sigma \sigma}$ from $[\overline{\mathrm{PT}}]$ may in the present case $(c=25, b=1)$ be simplified to

$$
g_{\beta}^{\sigma \sigma}=\mu_{r}^{\frac{\beta}{2}} \frac{\Gamma_{2}(2) \Gamma_{2}(2-2 \beta)}{\left(\Gamma_{2}(2-\beta)\right)^{2}} \frac{\Gamma_{2}(2 \sigma) \Gamma_{2}(4-2 \sigma)}{\Gamma_{2}(4-2 \sigma-\beta) \Gamma_{2}(2 \sigma-\beta)} .
$$

The function $\Gamma_{2}(x)$ is known as the Barnes Double Gamma function. It may be represented by the following integral [ [FZZ]:

$$
\log \Gamma_{2}(x)=\int_{0}^{\infty} \frac{d t}{t}\left(\frac{e^{-x t}-e^{-t}}{\left(1-e^{-t}\right)^{2}}-\frac{(1-x)^{2}}{2 e^{t}}-2 \frac{1-x}{t}\right) .
$$

It follows from (B.3) that $\Gamma_{2}(x)$ is analytic for $\operatorname{Re}(x)>0$.

\section{B.1 Bulk-boundary OPE}

Our first aim is to establish the relation (4.12) with $C_{\delta}(P)$ defined by $C_{\delta}(P) \equiv g_{\beta}^{\sigma \sigma}$. This relation is equivalent to the relation

$$
\Psi_{2 \alpha}^{\sigma \sigma}(\sigma)\left|B_{\delta}\right\rangle_{\mathrm{L}} \equiv \lim _{\tau \rightarrow 0}(2 \tau)^{2 \Delta_{\alpha}-\Delta_{2 \alpha}} V_{\alpha}(\tau, \sigma)\left|B_{\delta}\right\rangle_{\mathrm{L}}
$$

This relation will be valid as long as $-1<\delta<0$ and $\operatorname{Re}(\alpha)<\frac{1}{2}$. It can be used as a definition of the boundary fields $\Psi_{\beta}^{\sigma \sigma}(\sigma)$ for general values of $\sigma$ and $\beta$ thanks to the analyticity of the fields $\Psi_{\beta}^{\sigma \sigma}(\sigma)$ w.r.t. these variables [T2].

Our starting point for the derivation of (4.12) is the form of the bulk-boundary OPE valid for bulk fields $V_{\alpha}(z, \bar{z})$ which approach the boundary if $-1<\delta<0$ and $\frac{3}{2}>\operatorname{Re}(\alpha)>\frac{1}{2}$,

$$
V_{\alpha}(z, \bar{z})=\int_{\mathbb{S}} d \beta|z-\bar{z}|^{\Delta_{\beta}-2 \Delta_{\alpha}} A_{\alpha \mid \delta}^{\beta} \Psi_{\beta}^{\delta}(x)+(\text { descendants })
$$

where $\mathbb{S}=1+i \mathbb{R}_{+}$and $x=\operatorname{Re}(z)$. The coefficients $A_{\alpha \mid \delta}^{\beta}$ which appear in the bulk-boundary OPE are related to the expectation value

$$
A_{\beta \mid \alpha}^{\sigma}=\lim _{2 z \rightarrow i} \lim _{x \rightarrow \infty}|x|^{2 \Delta_{\beta}}\left\langle\Psi_{\beta}^{\sigma \sigma}(x) V_{\alpha}(z, \bar{z})\right\rangle_{\mathrm{L}, \delta}^{\mathbb{U}}
$$


via $A_{\delta \mid \alpha}^{\beta}=A_{Q-\beta \mid \alpha}^{\sigma}$, as follows by inserting (B.5) into (B.6) and taking into account that the fields $\Psi_{\beta}^{\sigma \sigma}(x)$ are normalized by $\lim _{x \rightarrow \infty}|x|^{2 \Delta_{\beta}}\left\langle\Psi_{\beta_{2}}^{\sigma \sigma}(x) \Psi_{\beta_{1}}^{\sigma \sigma}(0)\right\rangle_{\mathrm{L}, \delta}^{\mathbb{U}}=\delta\left(\beta_{2}-\beta_{1}\right)$ for $\beta_{2}, \beta_{1} \in \mathbb{S}$. The explicit expression for $A_{\beta \mid \alpha}^{\sigma}$ was found in [으. It may be represented as

$$
\begin{aligned}
& A_{\beta \mid \alpha}^{\sigma}=\rho_{\beta \mid \alpha} \int_{-\infty}^{\infty} d t \prod_{\epsilon= \pm} \frac{S_{2}\left(\frac{1}{2}(2 \alpha+\beta-2)+i \epsilon t\right)}{S_{2}\left(\frac{1}{2}(2 \alpha-\beta+2)+i \epsilon t\right)} e^{4 \pi t(\sigma-1)}, \\
& \text { where } \rho_{\beta \mid \alpha}=\mu^{\frac{2-2 \alpha-\beta}{2}} \frac{\Gamma_{2}^{3}(2-\beta) \Gamma_{2}(4-2 \alpha-\beta) \Gamma_{2}(2 \alpha-\beta)}{\Gamma_{2}(2) \Gamma_{2}(\beta) \Gamma_{2}(2-2 \beta) \Gamma_{2}(2 \alpha) \Gamma_{2}(2-2 \alpha)}
\end{aligned}
$$

Additional discrete terms will appear in the bulk-boundary OPE (B.5) as soon as $\operatorname{Re}(\alpha)<\frac{1}{2}$. There is a single discrete term proportional to $\Psi_{2 \alpha}(x)$ as long as $0<\operatorname{Re}(\alpha)<\frac{1}{2}$. In order to identify this contribution let us note that the OPE coefficients $A_{\delta \mid \alpha}^{\beta}$ have a pole near $\beta=2 \alpha$,

$$
A_{\delta \mid \alpha}^{\beta} \underset{\beta \rightarrow 2 \alpha}{\sim} \frac{1}{2 \pi} \frac{1}{2 \alpha-\beta} .
$$

This is shown by noting that contour of the integration in (B.7) gets pinched by the two poles of the integrand at $t= \pm \frac{i}{2}(2 \alpha-\beta)$. Extracting the resulting singular contribution to the integral by contour deformation yields (B.8).

Without loss of generality 7 let us assume that $\operatorname{Im}(\alpha)>0$. The pole at $\beta=2 \alpha$ would cross the contour of integration over $\beta$ in (B.5) when varying $\alpha$ from $\operatorname{Re}(\alpha)>\frac{1}{2}$ to $\operatorname{Re}(\alpha)<\frac{1}{2}$. The analytic continuation of the integral in (B.5) can be described by integrating over a deformed contour which is the sum of $\mathbb{S}$ with a small circle around the pole at $\beta=2 \alpha$. Evaluating the contribution from the latter by using the residue theorem and (B.8) leads to the conclusion that in the range $0<\operatorname{Re}(\alpha)<\frac{1}{2}$ the OPE (B.5) gets modified to

$$
\begin{array}{rl}
V_{\alpha}(z, \bar{z})=\int_{\mathbb{S}} & d \beta|z-\bar{z}|^{\Delta_{\beta}-2 \Delta_{\alpha}} A_{\alpha \mid \delta}^{\beta} \Psi_{\beta}^{\delta}(x) \\
& +|z-\bar{z}|^{\Delta_{2 \alpha}-2 \Delta_{\alpha}} \Psi_{2 \alpha}^{\delta}(x)+(\text { descendants })
\end{array}
$$

The discrete contribution in the second line of $(\overline{B .9})$ is the dominant one for $\operatorname{Im}(z) \rightarrow 0$. The sought-for relation (B.4) therefore follows directly from (B.9).

Let us remark that the functional equation satisfied by $\Gamma_{2}(x)$,

$$
\Gamma_{2}(x+1)=\sqrt{2 \pi}(\Gamma(x))^{-1} \Gamma_{2}(x) .
$$

together with the analyticity of $\Gamma_{2}(x)$ for $\operatorname{Re}(x)>0$ imply that $\Gamma_{2}(x)$ has a simple pole at $x=0$. This implies in particular that $C_{\delta}(i \delta)=g_{2 \sigma}^{\sigma \sigma}=0$, as is easily seen from (B.2).

It remains to derive the expression (4.18). Let us first observe that due to $\left\langle\left. P\right|_{\mathrm{L}}=\right.$ $\lim _{z \rightarrow \infty}|z|^{4 \Delta_{\alpha}}\left\langle\left. 0\right|_{\mathrm{L}} V_{Q-\alpha}(z, \bar{z})\right.$ for $\alpha=1+i P$ we have

$$
\left\langle P_{1}\left|\Phi_{P_{2}}^{\delta}(1)\right| B_{\delta}\right\rangle_{\mathrm{L}}=g_{1+i P_{2}}^{\sigma \sigma} A_{1+i P_{2} \mid 1-i P_{1}}^{\sigma} .
$$

\footnotetext{
${ }^{7}$ The other case leads to the same result thanks to the symmetry of the integrand in (B.5) under $\beta \rightarrow 2-\beta$.
} 
Note furthermore that for $\beta=1+i P_{2}, P_{2}=\mathcal{O}(\delta)$ we have $\beta-2 \sigma=\delta+i P_{2} \rightarrow 0$. Formula (B.13) of [T2] is therefore applicable in the case at hand and gives

$$
A_{1+i P_{2} \mid 1-i P_{1}}^{\sigma} \underset{\delta \rightarrow 0}{\sim} \frac{\mu_{r}^{\frac{1}{2}}}{2 \pi} \frac{2 P_{2}}{P_{2}-i \delta} \frac{P_{1}}{\sinh 2 \pi P_{1}} \Theta(P) .
$$

Equation (4.18) now follows easily by inserting (B.12) and (B.19) into (B.11).

\section{B.2 Structure functions of boundary fields}

It follows from (B.1) that $F_{P_{2} P_{1}}^{P_{3}}$ and $D_{P_{3} P_{2} P_{1}}$ can be expressed in terms of the OPE coefficients $C_{\alpha_{3} \mid \alpha_{2} \alpha_{1}}^{\sigma \sigma \sigma}$ and the three point functions $C_{\alpha_{3} \alpha_{2} \alpha_{1}}^{\sigma \sigma \sigma} \equiv C_{Q-\alpha_{3} \mid \alpha_{2} \alpha_{1}}^{\sigma \sigma \sigma}$ of the fields $\Psi_{\beta}^{\sigma_{2} \sigma_{1}}(x)[\overline{\mathrm{PT}}]$,

$$
D_{P_{3} P_{2} P_{1}}=g_{\alpha_{3}}^{\sigma \sigma} g_{\alpha_{2}}^{\sigma \sigma} g_{\alpha_{1}}^{\sigma \sigma} C_{\alpha_{3} \alpha_{2} \alpha_{1}}^{\sigma \sigma \sigma}, \quad F_{P_{2} P_{1}}^{P_{3}}=\frac{g_{\alpha_{2}}^{\sigma \sigma} g_{\alpha_{1}}^{\sigma \sigma}}{g_{\alpha_{3}}^{\sigma \sigma}} C_{\alpha_{3} \mid \alpha_{2} \alpha_{1}}^{\sigma \sigma \sigma}
$$

The formula for $C_{\alpha_{3} \mid \alpha_{2} \alpha_{1}}^{\sigma \sigma \sigma}$ from [PT] simplifies slightly in the present case,

$$
\begin{aligned}
C_{\alpha_{3} \mid \alpha_{2} \alpha_{1}}^{\sigma \sigma \sigma}= & \mu_{r}^{\frac{i}{2}\left(P_{3}-P_{2}-P_{1}\right)} \Gamma_{2}\left(1-i\left(P_{1}+P_{2}+P_{3}\right)\right) \\
& \times \frac{\Gamma_{2}\left(1+i\left(P_{2}+P_{3}-P_{1}\right)\right) \Gamma_{2}\left(1+i\left(P_{2}-P_{1}-P_{3}\right)\right) \Gamma_{2}\left(1+i\left(P_{3}-P_{\mathbf{1}}-P_{2}\right)\right)}{\Gamma_{2}\left(2 i P_{3}\right) \Gamma_{2}\left(-2 i P_{2}\right) \Gamma_{2}\left(-2 i P_{\mathbf{1}}\right) \Gamma_{2}(2)} \\
& \times \frac{S_{2}\left(1+i P_{3}\right) S_{2}\left(2+\delta+i P_{3}\right)}{S_{2}\left(1+i P_{2}\right) S_{2}\left(2+\delta+i P_{2}\right)} \int_{\mathbb{R}+i 0} d s \prod_{k=1}^{4} \frac{S_{2}\left(U_{k}+i s\right)}{S_{2}\left(V_{k}+i s\right)}
\end{aligned}
$$

where we have used the identifications from (B.1), the definitions $S_{2}(x)=\Gamma_{2}(x) / \Gamma_{2}(2-x)$ and

$$
\begin{array}{ll}
U_{1}=-\delta-i P_{1}, & V_{1}=2-i\left(P_{1}-P_{3}\right), \\
U_{2}=1-i P_{1}, & V_{2}=2-i\left(P_{1}+P_{3}\right), \\
U_{3}=1-i P_{2}, & V_{3}=1-\delta, \\
U_{4}=1+i P_{2}, & V_{4}=2 .
\end{array}
$$

Taking the limit $\delta \rightarrow 0, P_{k}=\mathcal{O}(\delta)$ within the formulae $(\mathrm{B} .13),(\mathrm{B} .14)$ is subtle due to the fact that many of the factors exhibit singular behavior. First note that it follows from the functional equation (B.10) that $\Gamma_{2}(x)$ behaves for $x \rightarrow 0$ as

$$
\Gamma_{2}(x) \underset{x \rightarrow 0}{\sim} \frac{1}{x} \frac{\Gamma_{2}(1)}{\sqrt{2 \pi}}
$$

Let us next consider the integral which appears in (B.14). We observe that the contour of integration in (B.14) gets pinched between the poles of the integrand at $i s=-U_{1}=\alpha_{1}-2 \sigma=$ $\delta\left(i q_{\mathrm{I}}-1\right)$ and $i s=2-V_{4}=0$. It follows that the integral diverges in this limit. The singular behavior for $\delta \rightarrow 0$ is identified by deforming the contour of integration into the contour $\mathbb{R}+i 0$ 
plus a small circle $\mathcal{C}_{1}$ around the pole at $s=i U_{1}$. The contribution from the latter is found to be

$$
\begin{aligned}
\int_{\mathcal{C}_{1}} d s \prod_{k=1}^{4} \frac{S_{2}\left(U_{k}+i s\right)}{S_{2}\left(V_{k}+i s\right)} \underset{\underset{\delta \rightarrow 0}{\sim}}{\sim} S_{2}\left(-\delta+i P_{3}\right) S_{2}\left(-\delta-i P_{3}\right) S_{b}\left(-\delta-i P_{\mathbf{1}}\right) \\
\underset{\delta \rightarrow 0}{\sim} \frac{1}{(2 \pi)^{3}} \frac{1}{-\delta+i P_{3}} \frac{1}{-\delta-i P_{3}} \frac{1}{-\delta-i P_{\mathbf{1}}}
\end{aligned}
$$

where we have used $S_{2}(x) S_{2}(2-x)=1$ and $S_{2}(x)=1$. Collecting the factors yields

$$
C_{\alpha_{3} \alpha_{2} \alpha_{1}}^{\sigma \sigma \sigma} \underset{\delta \rightarrow 0}{\sim} 2 \pi \frac{\mu_{r}^{-\frac{3}{2}}}{(2 \pi)^{3}} \prod_{k=1}^{3} \frac{2 P_{k}}{P_{k}+i \delta} .
$$

One finds similarly

$$
g_{\beta_{k}}^{\sigma \sigma} \underset{\delta \rightarrow 0}{\sim} 2 \pi \mu_{r}^{\frac{1}{2}} \frac{P_{k}-i \delta}{2 P_{k}} \quad k=1,2,3 .
$$

The approximate expressions for the three point functions $D_{P_{3} P_{2} P_{1}}$ and operator product coefficients $F_{P_{2} P_{1}}^{P_{3}}$ now follow easily by inserting ( $(\underline{\mathrm{B} .19})$ and $(\underline{\mathrm{B} .18)}$ into $(\underline{\mathrm{B} .13})$.

\section{B.3 A note on the normalizations}

The normalizations used in the present paper are fully fixed by the requirements

$$
\begin{aligned}
& \lim _{z_{2} \rightarrow z_{1}}\left|z_{2}-z_{1}\right|^{2\left(\Delta_{\alpha_{2}}+\Delta_{\alpha_{1}}-\Delta_{\alpha_{2}+\alpha_{1}}\right)} V_{\alpha_{2}}\left(z_{2}, \bar{z}_{2}\right) V_{\alpha_{1}}\left(z_{1}, \bar{z}_{1}\right)=V_{\alpha_{1}+\alpha_{2}}\left(z_{1}, \bar{z}_{1}\right), \\
& \lim _{x_{2} \rightarrow x_{1}}\left|x_{2}-x_{1}\right|^{\Delta_{\beta_{2}}+\Delta_{\beta_{1}}-\Delta_{\beta_{2}+\beta_{1}}} \Psi_{\beta_{2}}^{\sigma \sigma}\left(x_{2}\right) \Psi_{\beta_{1}}^{\sigma \sigma}\left(x_{1}\right)=\Psi_{\beta_{2}+\beta_{1}}^{\sigma \sigma}\left(x_{1}\right) \\
& \lim _{z-\bar{z} \rightarrow 0}|z-\bar{z}|^{2 \Delta_{\alpha}-\Delta_{2 \alpha}} V_{\alpha}(z, \bar{z})=\Psi_{2 \alpha}^{\sigma \sigma}(x)
\end{aligned}
$$

which follow from

$$
\begin{aligned}
& V_{\alpha}(z, \bar{z}) \underset{\phi_{0} \rightarrow-\infty}{\sim}: e^{2 \alpha \phi(z, \bar{z})}:, \\
& \Psi_{\beta}^{\sigma \sigma}(x) \underset{\phi_{0} \rightarrow-\infty}{\sim}: e^{\beta \phi(x)}: .
\end{aligned}
$$

In order to show that $(\underline{B .14})$ implies $(\mathrm{B} .21)$ one may proceed as in the discussion of the bulkboundary OPE in subsection B.1. Equation (B.21) follows from the observation that

$$
C_{\alpha_{3} \mid \alpha_{2} \alpha_{1}}^{\sigma \sigma \sigma} \underset{\alpha_{3} \rightarrow \alpha_{1}+\alpha_{2}}{\sim} \frac{1}{2 \pi} \frac{1}{\alpha_{1}+\alpha_{2}-\alpha_{3}}
$$

which is shown by the same method as $(\underline{B} .8)$. The normalization prescriptions used in [FZZ, Ho, T2] differ slightly from the one used in this paper. 


\section{References}

[FHL] D.Z. Freedman, M. Headrick, A. Lawrence, "On Closed String Tachyon Dynamics", Phys.Rev. D73 (2006) 066015 [arXiv:hep-th/0510126]

[GM] P. Ginsparg, G. Moore, "Lectures on 2D gravity and 2D string theory (TASI 1992)", in Recent Directions in Particle Theory, eds. J. Harvey and J. Polchinski, Proceedings of the 1992 TASI, World Scientific, Singapore, 1993

[SS] N. Seiberg, D. Shih, "Minimal string theory", Comptes Rendus Physique 6 (2005) 165174 [arXiv:hep-th/0409306]

[CL] A. Cappelli, J.I. Latorre, "Perturbation theory of higher-spin conserved currents off criticality”, Nucl. Phys. B340 (1990) 659-691

[FZZ] V. Fateev, A. B. Zamolodchikov and A. B. Zamolodchikov, "Boundary Liouville field theory. I: Boundary state and boundary two-point function," [arXiv:hep-th/0001012].

[T0] J. Teschner, "Liouville theory revisited," Class. Quant. Grav. 18, R153 (2001) [arXiv:hep-th/0104158].

[T1] J. Teschner, "Remarks on Liouville with a boundary", Talk at TMR-conference "Nonperturbative Quantum Effects 2000 [arXiv:hep-th/0009138].

[Ho] K. Hosomichi, "Bulk-Boundary Propagator in Liouville Theory on a Disc", JHEP 0111 (2001) 044

[ZZ] A. B. Zamolodchikov and A. B. Zamolodchikov, "Structure constants and conformal bootstrap in Liouville field theory," Nucl. Phys. B 477, 577 (1996) [arXiv:hep-th/9506136].

[PT] B. Ponsot and J. Teschner, "Boundary Liouville field theory: Boundary three point function,” Nucl. Phys. B 622, 309 (2002) [arXiv:hep-th/0110244].

[T2] J. Teschner, "On boundary perturbations in Liouville theory and brane dynamics in noncritical string theory", JHEP 04 (2004) 023

[DIR] A. Dabholkar, A. Iqubal, J. Raeymaekers, "Off-Shell Interactions for Closed-String Tachyons”, JHEP 0405 (2004) 051 [arXiv:hep-th/0403238]

[SarSat] S. Sarkar, B. Sathiapalan, "Closed String Tachyons on $C / Z_{N}$ ", Int.J.Mod.Phys. A19 (2004) 5625-5638 [arXiv:hep-th/0309029]

[SeibSh] N. Seiberg, S. Shenker, “A Note on Background (In)dependence”, Phys.Rev. D45 (1992) 4581-4587 [arXiv:hep-th/9201017] 
[APS] A. Adams, J. Polchinski, E. Silverstein, "Don't Panic! Closed String Tachyons in ALE Spacetimes", JHEP 0110 (2001) 029 [arXiv:hep-th/0108075] 